\title{
Summary of TRUEX Radiolysis Testing Using the INL Radiolysis Test Loop
}

Dean R. Peterman

Lonnie G. Olson

Rocklan G. McDowell

Gracy Elias Jack D. Law

March 2012

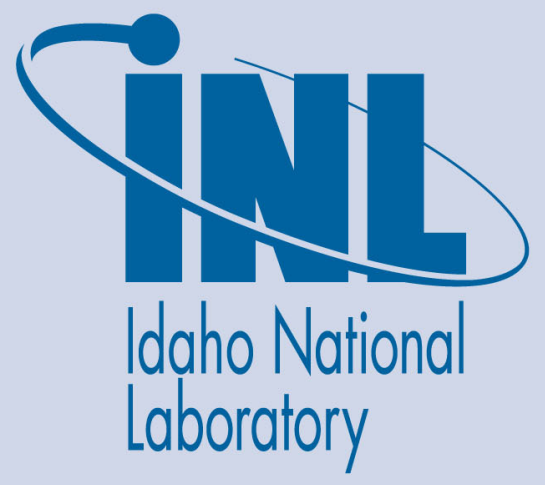

The INL is a U.S. Department of Energy National Laboratory operated by Battelle Energy Alliance 
INL/EXT-12-25311

FCRD-SWF-2012-000082

\title{
Summary of TRUEX Radiolysis Testing Using the INL Radiolysis Test Loop
}

\author{
Dean R. Peterman \\ Lonnie G. Olson \\ Rocklan G. McDowell \\ Gracy Elias \\ Jack D. Law
}

March 2012

\author{
Idaho National Laboratory \\ Fuel Cycle Research \& Development \\ Idaho Falls, Idaho 83415
}

http://www.inl.gov

Prepared for the

U.S. Department of Energy

Office of Nuclear Energy

Under DOE Idaho Operations Office

Contract DE-AC07-05ID14517 


\section{DISCLAIMER}

This information was prepared as an account of work sponsored by an agency of the U.S. Government. Neither the U.S. Government nor any agency thereof, nor any of their employees, makes any warranty, expressed or implied, or assumes any legal liability or responsibility for the accuracy, completeness, or usefulness, of any information, apparatus, product, or process disclosed, or represents that its use would not infringe privately owned rights. References herein to any specific commercial product, process, or service by trade name, trade mark, manufacturer, or otherwise, does not necessarily constitute or imply its endorsement, recommendation, or favoring by the U.S. Government or any agency necessarily state or reflect those of the U.S. Government or any agency 


\section{SUMMARY}

The INL radiolysis and hydrolysis test loop has been used to evaluate the effects of hydrolytic and radiolytic degradation upon the efficacy of the TRUEX flowsheet for the recovery of trivalent actinides and lanthanides from acidic solution. The results demonstrate that solvent hydrolysis does not adversely impact the performance of the process flowsheet.

The nominal composition of the TRUEX solvent used in this study is $0.2 \mathrm{M} \mathrm{CMPO}$ and $1.4 \mathrm{M}$ TBP dissolved in n-dodecane. Gamma irradiation of a mixture of TRUEX process solvent and nitric acid in the test loop does adversely impact flowsheet performance as measured by the decreasing americium and europium distribution ratios in the extraction section as accumulated dose increases. The observed increase in americium and europium stripping distribution ratio with increasing absorbed dose indicates the radiolytic production of organic soluble degradation compounds. These organic soluble degradation products inhibit stripping of the irradiated TRUEX solvent with dilute nitric acid. Using GC, IC and HPLC, the changes in the TRUEX solvent composition were quantified. The changes in the TRUEX solvent composition correlate will with variation in the measured extraction, scrub, and strip Am and Eu distribution ratios.

Operation of the TRUEX flowsheet would require careful monitoring to ensure extraction distributions are maintained at acceptable levels. Results of flowsheet testing demonstrate that acidic degradation products are removed from the solvent. Non-acidic degradation products build-up in the process solvent but these products do not adversely impact stripping performance. Use of an aqueous complexant in the aqueous strip solution easily overcomes the adverse impacts of non-acidic degradation and maintains very efficient stripping of the TRUEX process solvent at all absorbed doses studied. 


\section{CONTENTS}

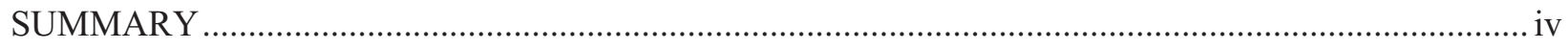

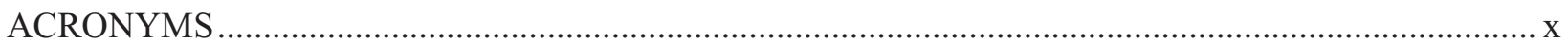

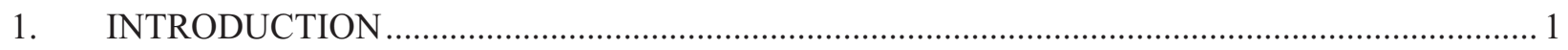

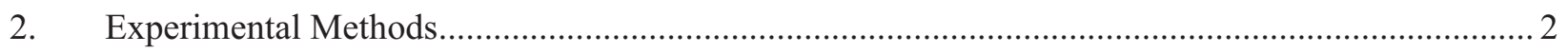

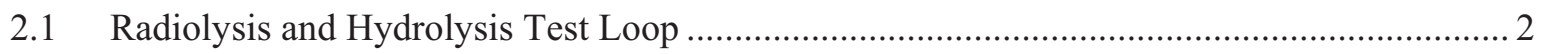

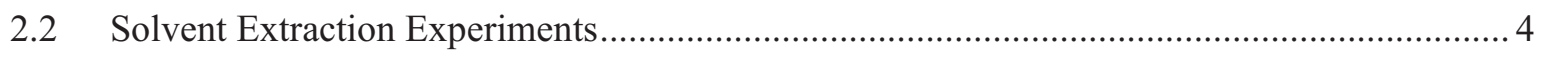

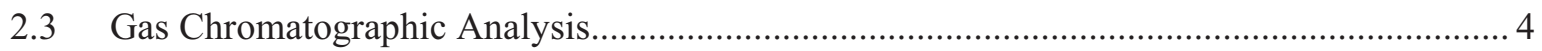

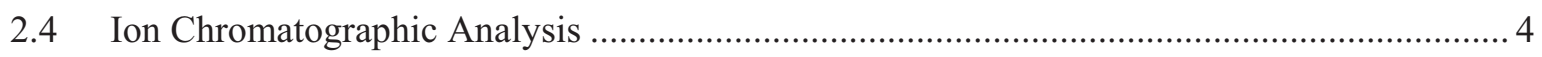

2.5 High Performance Liquid Chromatographic Analysis.................................................... 5

3. Results - Hydrolysis and Radiolysis Experiments in Test Loop................................................. 5

3.1 Solvent Extraction Experiments....................................................................... 7

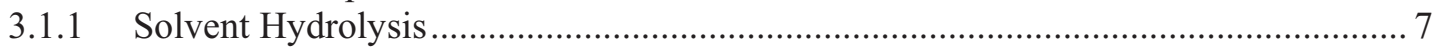

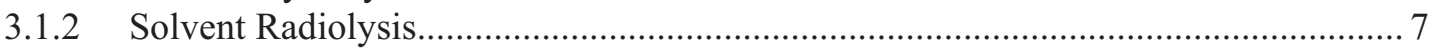

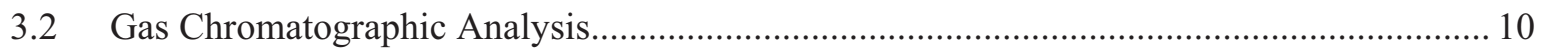

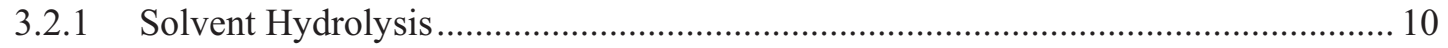

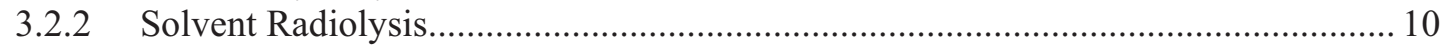

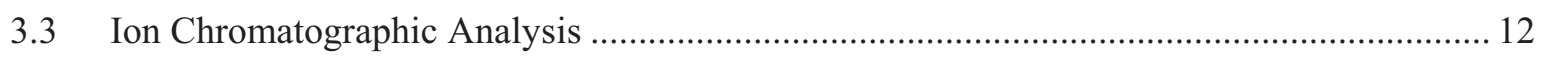

3.4 High Performance Liquid Chromatographic Analysis................................................... 16

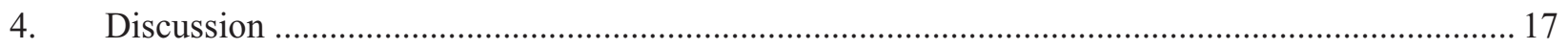

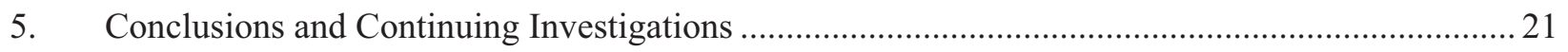

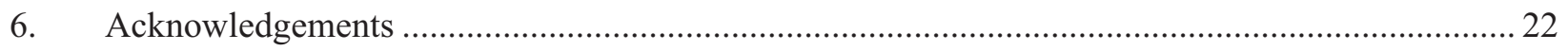

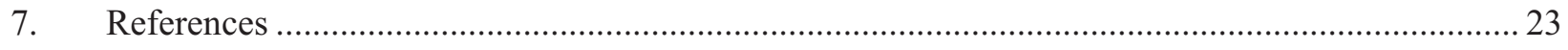




\section{FIGURES}

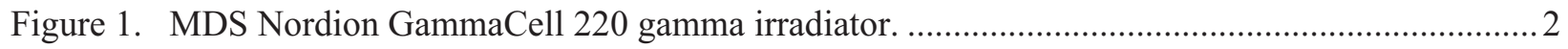

Figure 2. Schematic diagram of the INL Radiolysis and Hydrolysis Test Loop. ..................................... 3

Figure 3. Concentration of $\mathrm{HNO}_{3}$ present in the aqueous phase versus absorbed dose for nitric acid in contact with TRUEX solvent. Diamond data markers: determined acid concentration.

Figure 4. Concentration of $\mathrm{HNO}_{3}$ present in the organic phase versus absorbed dose for TRUEX solvent in contact with nitric acid.

Figure 5. Measured extraction distribution ratios of Am (open diamonds) and Eu (open squares) for TRUEX solvent irradiated in the INL TEST loop in contact with $4.4 \mathrm{M} \mathrm{HNO}_{3}$ as a function of absorbed dose.

Figure 6. Measured stripping distribution ratios of Am (open diamonds) and Eu (open squares) for TRUEX solvent irradiated in the INL TEST loop in contact with $4.4 \mathrm{M} \mathrm{HNO}_{3}$ as a function of absorbed dose.

Figure 7. Typical GC-FID chromatogram for TBP analysis. Retention times: $\mathrm{RT}=6.5 \mathrm{~min}, \mathrm{n}-$ dodecane; $\mathrm{RT}=8.5 \mathrm{~min}, 2$-dodecanol; $\mathrm{RT}=10.4 \mathrm{~min}, \mathrm{TBP}$.

Figure 8. Typical GC-FID chromatogram for $\mathrm{HDBP}$ and $\mathrm{H}_{2} \mathrm{MBP}$ analysis. Retention times: RT $=7.6 \mathrm{~min}$, methyl ester of $\mathrm{H}_{2} \mathrm{MBP} ; \mathrm{RT}=9.2 \mathrm{~min}$, methyl ester of HDBP.

Figure 9. Concentration of TBP determined by GC-FID analysis present in the irradiated TRUEX solvent as a function of absorbed dose.

Figure 10. Concentration of HDBP determined by GC-FID analysis present in the irradiated TRUEX solvent as a function of absorbed dose.

Figure 11. Ion chromatograph of aqueous nitric acid phase following irradiation to an absorbed dose of $785 \mathrm{kGy}$. Peak eluting between 5 - $10 \mathrm{~min}$ due to nitrate anion.

Figure 12. Ion chromatograph of aqueous nitric acid phase following irradiation to an absorbed dose of $785 \mathrm{kGy}$ - expanded y-axis. Retention times: $\mathrm{RT}=2.5 \mathrm{~min}$, unknown; $\mathrm{RT}=$ $4 \mathrm{~min}$, anion of HDBP; $\mathrm{RT}=5-10 \mathrm{~min}$, nitrate anion; $\mathrm{RT}=12 \mathrm{~min}$, anion of $\mathrm{H}_{2} \mathrm{MBP}$; $\mathrm{RT}=15.5-17.5 \mathrm{~min}$, unknown; $\mathrm{RT}=22.5 \mathrm{~min}$, phosphate anion.

Figure 13. Concentration of HDBP determined by IC analysis present in the irradiated $\mathrm{HNO}_{3}$ as a function of absorbed dose.

Figure 14. Concentration of $\mathrm{H}_{2} \mathrm{MBP}$ determined by IC analysis present in the irradiated $\mathrm{HNO}_{3}$ as a function of absorbed dose.

Figure 15. Concentration of phosphate anion determined by IC analysis present in the irradiated $\mathrm{HNO}_{3}$ as a function of absorbed dose.

Figure 16. Plot of peak area versus absorbed dose for two unidentified products of TRUEX radiolysis determined by IC analysis of the irradiated 4.4 $\mathrm{M} \mathrm{HNO}_{3}$. Retention times: $2.5 \mathrm{~min}$ (diamonds) and $16.8 \mathrm{~min}$ (squares).

Figure 17. Typical HPLC chromatogram for the analysis of TRUEX solvent. Retention times: $\mathrm{RT}=7.5 \mathrm{~min}$, triphenyl phosphate (used as internal standard); RT = $15.0 \mathrm{~min}, \mathrm{CMPO}$. .16 
Figure 18. Concentration of CMPO determined by HPLC analysis present in the irradiated TRUEX solvent as a function of absorbed dose.

Figure 19. Plot of peak area versus absorbed dose for three unidentified products of TRUEX radiolysis determined by HPLC analysis of the irradiated TRUEX solvent. Retention times: $5.8 \mathrm{~min}$ (circles), $8.8 \mathrm{~min}$ (squares) and $13 \mathrm{~min}$ (diamonds).

Figure 20. Plot of the concentration of TBP versus absorbed dose for TRUEX solvent irradiated in contact with $4.4 \mathrm{M} \mathrm{HNO}_{3}$. The slope of the linear best-fit line corresponds to the $G$ value for the decomposition of TBP. The determined $G$-value for the destruction of $\mathrm{TBP}$ is $-G_{\mathrm{TBP}}=-0.114 \pm 0.031 \mu \mathrm{mol} \cdot \mathrm{L}^{-1} \cdot \mathrm{Gy}^{-1} .\left(\mathrm{R}^{2}=0.62\right)$

Figure 21. Plot of the concentration of CMPO versus absorbed dose for TRUEX solvent irradiated in contact with $4.4 \mathrm{M} \mathrm{HNO}_{3}$. The slope of the linear best-fit line corresponds to the $G$-value for the decomposition of CMPO. The determined $G$-value for the destruction of CMPO is $-G_{\mathrm{CMPO}}=-0.159 \pm 0.016 \mu \mathrm{mol} \cdot \mathrm{L}^{-1} \cdot \mathrm{Gy}^{-1} .\left(\mathrm{R}^{2}=0.97\right)$.

Figure 22. Plot of the total concentration of HDBP present in the aqueous and organic phases versus absorbed dose for TRUEX solvent irradiated in contact with $4.4 \mathrm{M} \mathrm{HNO}_{3}$. The slope of the linear best-fit line corresponds to the $G$-value for the production of HDBP. The determined $G$-value for the overall production of HDBP is $G_{\mathrm{HDBP}}=0.118 \pm 0.007$ $\mu \mathrm{mol} \cdot \mathrm{L}^{-1} \cdot \mathrm{Gy}^{-1}$. $\left(\mathrm{R}^{2}=0.97\right)$

Figure 23. Plot of the concentration of $\mathrm{H}_{2} \mathrm{MBP}$ present in the aqueous phase versus absorbed dose for TRUEX solvent irradiated in contact with $4.4 \mathrm{M} \mathrm{HNO}_{3}$. The slope of the linear best-fit line corresponds to the $G$-value for the production of $\mathrm{H}_{2} \mathrm{MBP}$. The determined $G$-value for the production of $\mathrm{H}_{2} \mathrm{MBP}$ is $G_{\mathrm{H} 2 \mathrm{MBP}}=1.56 \times 10^{-3} \pm 8.06 \times 10^{-5}$ $\mu \mathrm{mol} \cdot \mathrm{L}^{-1} \cdot \mathrm{Gy}^{-1} \cdot\left(\mathrm{R}^{2}=0.99\right)$

Figure 24. Plot of the concentration of phosphate anion present in the aqueous phase versus absorbed dose for TRUEX solvent irradiated in contact with $4.4 \mathrm{M} \mathrm{HNO}_{3}$. The slope of the linear best-fit line corresponds to the $G$-value for the production of phosphate anion. The determined $G$-value for the production of phosphate is $G_{\mathrm{PO} 4-3}=5.60 \times 10^{-3} \pm$ $3.94 \times 10^{-4} \mu \mathrm{mol} \cdot \mathrm{L}^{-1} \cdot \mathrm{Gy}^{-1}$. $\left(\mathrm{R}^{2}=0.98\right)$ 


\section{TABLES}

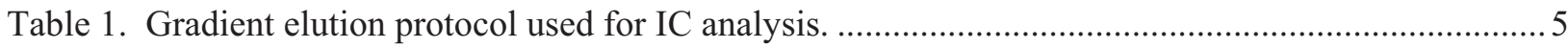

Table 2. Am distribution ratios for TRUEX solvent irradiated in contact with $4.4 \mathrm{M} \mathrm{HNO}_{3}$ as a

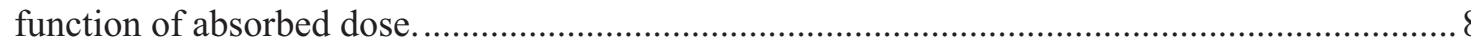

Table 3. Eu distribution ratios for TRUEX solvent irradiated in contact with $4.4 \mathrm{M} \mathrm{HNO}_{3}$ as a

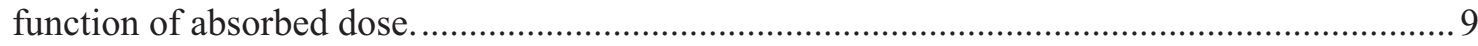

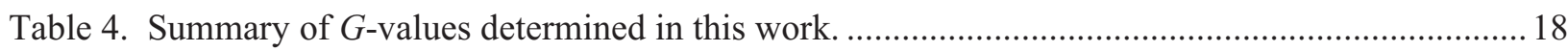




\section{ACRONYMS}

\begin{tabular}{|c|c|}
\hline AMUSE & Argonne Model for Universal Solvent Extraction \\
\hline CINC & Costner Industries Nevada Corporation \\
\hline GC-FID & Gas chromatography with flame ionization detection \\
\hline CMPO & Octyl(phenyl)-N, N-diisobutylcarbamoylmethyl phosphine oxide \\
\hline DTPA & Diethylenetriaminepentaacetic acid \\
\hline ESI-MS & Electrospray ionization mass spectrometry \\
\hline HDBP & Dibutylphosphoric acid \\
\hline $\mathrm{H}_{2} \mathrm{MBP}$ & Monobutylphosphoric acid \\
\hline HPLC & High performance liquid chromatography \\
\hline IC & Ion chromatography with conductivity detection \\
\hline INL & Idaho National Laboratory \\
\hline $\mathrm{O} / \mathrm{A}$ & Organic to aqueous phase ratio \\
\hline PUREX & Plutonium Uranium Reduction EXtraction \\
\hline TBP & Tri- $n$-butylphosphate \\
\hline TPP & Triphenylphosphate \\
\hline TRUEX & TRansUranic EXtraction \\
\hline
\end{tabular}





\section{SEPARATIONS CAMPAIGN}

\section{SUMMARY OF TRUEX RADIOLYSIS TESTING USING THE INL RADIOLYSIS TEST LOOP}

\section{INTRODUCTION}

The radiolysis/hydrolysis test loop, located at the Idaho National Laboratory (INL), was utilized to study the impacts of radiolytic and hydrolytic degradation products on the performance of the TRansUranic EXtraction (TRUEX) process. The TBP/alkane diluent system has received extensive attention in the scientific literature for at least the last forty years. The radiation chemistry of the TBP was recently reviewed by Mincher and co-authors. ${ }^{1}$ Further, Mincher et al. have also recently reviewed the radiation chemistry of organophosphorus extractants such as octylphenyl-N,N-diisobutylcarbamoylmethyl phosphine oxide (CMPO), one of the components of the TRUEX process solvent. ${ }^{2}$

The successful deployment of any solvent extraction ligand proposed for use in fuel cycle separations will depend upon the stability of that ligand in an acidic, radioactive environment. Irradiation of the ligand occurs due to the decay energy of actinides and fission products in the dissolved nuclear fuel solution. The radiation types are predominantly low linear energy transfer (LET) beta/gamma radiation from fission product decay, and high LET alpha radiation from actinide decay. The major reactive species formed $^{3,4}$ by radiolysis of water, alkane diluent, and nitric acid are shown in Equations 1 - 3, respectively.

$$
\begin{aligned}
& \mathrm{H}_{2} \mathrm{O}-\mathrm{M} \rightarrow{ }^{\circ} \mathrm{OH}+\mathrm{e}^{-}+\mathrm{H}^{\cdot}+\mathrm{H}_{2} \mathrm{O}_{2}+\mathrm{H}_{3} \mathrm{O}^{+}+\mathrm{H}_{2} \\
& \mathrm{CH}_{3}\left(\mathrm{CH}_{2}\right)_{\mathrm{n}} \mathrm{CH}_{3}-\mathrm{M} \rightarrow \mathrm{e}^{-}{ }_{\text {sol }}+\mathrm{CH}_{3}\left(\mathrm{CH}_{2}\right)_{\mathrm{n}} \mathrm{CH}_{3}{ }^{+}+\mathrm{CH}_{3}\left(\mathrm{CH}_{2}\right)_{\mathrm{n}} \mathrm{CH}_{2} \cdot+{ }^{\circ} \mathrm{CH}_{3}+\mathrm{H}^{\cdot}+\mathrm{H}_{2} \\
& \mathrm{HNO}_{3}-\mathrm{W} \rightarrow \mathrm{NO}_{2}+{ }^{\circ} \mathrm{NO}_{3}+, \mathrm{HNO}_{2}
\end{aligned}
$$

Equations $1-3$ show that radiolysis of aqueous and organic phases generates a range of oxidizing ( ${ }^{\circ} \mathrm{OH}$, $\left.\mathrm{NO}_{3}, \mathrm{NO}_{2}\right)$ radicals, reducing $\left(\mathrm{H}^{*}\right)$ radicals, the reducing aqueous electron $\left(\mathrm{e}^{-}{ }_{\mathrm{aq}}\right)$, and reactive molecular species $\left(\mathrm{H}_{2} \mathrm{O}_{2}, \mathrm{HNO}_{2}, \mathrm{H}_{2}\right)$.

Due to relatively low ligand concentrations employed most decay energy is absorbed by the diluent and ligand radiolysis is expected to occur by indirect, rather than direct mechanisms. ${ }^{5}$ For example, the proposed TRUEX solvent contains 0.2 M CMPO as the ligand, while the tributylphosphate (TBP) modifier and dodecane diluent concentrations are about 1.4 M and $3 \mathrm{M}$, respectively. Consequently, most ligand damage will be due to reaction with reactive species created by energy deposition in the balance of the organic phase, or in the acidic aqueous phase in contact with that organic phase. Therefore, realistic examination of the impacts of radiolytic degradation upon the efficacy of solvent extraction processes used in an advanced nuclear fuel cycle, necessitates studying the radiolysis of mixed aqueous and organic systems. 


\section{Experimental Methods}

\subsection{Radiolysis and Hydrolysis Test Loop}

The design, construction, and installation of the solvent radiolysis and hydrolysis test loop have been described previously. ${ }^{6}$ The irradiation source (see Figure 1) is a MDS Nordion GammaCell 220 Excel self-contained ${ }^{60} \mathrm{Co}$ gamma irradiator. The center-line gamma dose rate in the sample chamber is approximately $6.5 \mathrm{kGy} / \mathrm{hr}$. The solvent irradiation loop is based upon a coil of borosilicate glass tubing ( 0.375 " OD, 0.202" ID) which is placed in the gamma irradiator sample chamber. The current effective gamma dose rate in the test loop is 3.5 $\mathrm{kGy} / \mathrm{hr}$. During the solvent irradiation, the

aqueous and organic phases are mixed using a centrifugal contactor (CINC V-02, USA) with

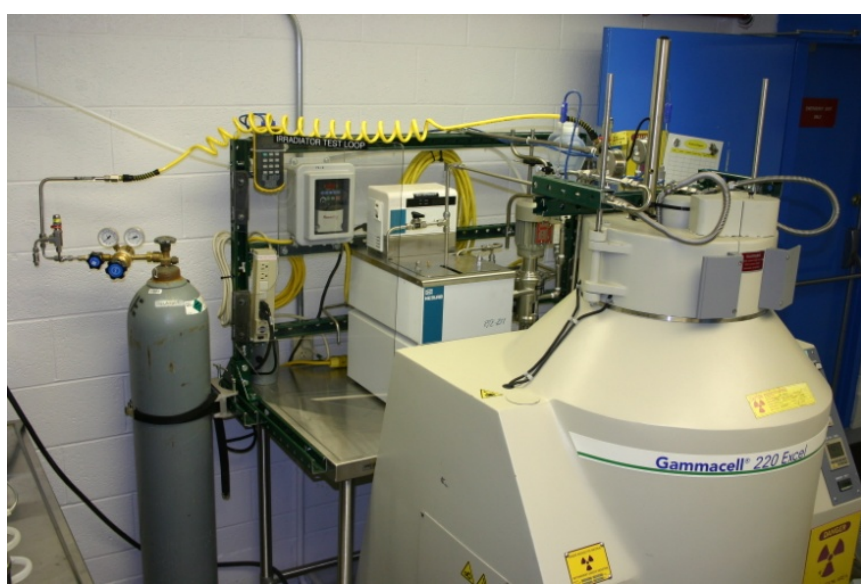

Figure 1. MDS Nordion GammaCell 220 gamma irradiator. the rotor replaced by a four vane mixing paddle.

The interior of the centrifugal contactor housing may be purged with inert gas, if necessary. The organic and aqueous phases used are metered into the mixing region of the contactor at the desired organic to aqueous phase ratio $(\mathrm{O} / \mathrm{A})$. The mixed phases are pumped through the irradiator test loop by a magnetic drive gear pump. The mixed phases flow through the glass coil in the irradiator sample chamber, through an external coil in a water bath, and return to the inlet of the centrifugal contactor where the phases are mixed and circulated back through the loop. In-line tube mixers (TAH Industries, Inc.) are used to provide additional phase mixing in the test loop. The in-line tube mixers are placed between the outlet of the gear drive pump and the inlet of the irradiation loop and between the outlet of the irradiation loop and the inlet to the external temperature control coil. The flowrate of the dispersion is in the range of 1.5 $\mathrm{L} / \mathrm{min}$ in order to maintain turbulent flow and keep the phases dispersed. The radiolysis/hydrolysis test loop is equipped with pressure gauges and thermocouples before and after the gamma irradiator. Two flow sight glasses are used to monitor the extent of mixing in the test loop. The two phases continue to circulate until the desired dose is obtained.

The temperature inside the sample chamber is $\sim 38^{\circ} \mathrm{C}$ due to the decay heat of the ${ }^{60} \mathrm{Co}$ source. Lower temperature irradiations are possible by using the off-gas of a liquid nitrogen dewar and a control valve interfaced to a temperature controller to maintain a temperature below $38{ }^{\circ} \mathrm{C}$ in the sample chamber. The lower limit for the operating temperature has not been determined, but irradiations at $10{ }^{\circ} \mathrm{C}$ are possible. A temperature-controlled water bath (external to the sample chamber) is used to maintain the desired temperature of the test solutions outside of the irradiator sample chamber. The sampling port permits samples to be withdrawn from the test loop in order to monitor the conditions during an irradiation. To facilitate hydrolysis of the solvent in the test loop, this setup will allow the solution to be maintained at an elevated temperature throughout testing if desired. A schematic view of the radiolysis/hydrolysis test loop is shown in Figure 2. 
Figure 2. Schematic diagram of the INL Radiolysis and Hydrolysis Test Loop. 


\subsection{Solvent Extraction Experiments}

In order to gauge the effect of gamma radiolysis upon the extraction performance of the TRUEX solvent used in this study, americium and europium distribution ratios were measured as a function of absorbed dose. All chemicals were reagent grade or higher (Sigma Aldrich). Radiotracers $\left({ }^{241} \mathrm{Am}\right.$ and $\left.{ }^{152,154} \mathrm{Eu}\right)$ were obtained from laboratory stocks. Aqueous solutions were prepared using de-ionized water. All analyses are performed in triplicate unless otherwise noted.

A simplified extraction/scrub/strip flowsheet (not shown) was employed to evaluate the effect of gamma radiolysis on the TRUEX process. The distribution of the americium and europium radiotracers was determined by calculating the distribution ratio, $\mathrm{D}=[\mathrm{M}]$ org/ $[\mathrm{M}] \mathrm{aq}$. The concentration of radiotracers present in the organic and aqueous phases was determined by gamma spectrometry. All flowsheet contacts were performed in triplicate as a function of absorbed dose. The organic to aqueous phase volume ratio $(\mathrm{O} / \mathrm{A})$ in the extraction, scrub, and strip sections of the flowsheet were 1.0, 0.2, and 0.1, respectively. The aqueous phase used in the extraction section was irradiated nitric acid with added radiotracers. The compositions of the aqueous phase used in scrub, strip sections were $1.37 \mathrm{M} \mathrm{HNO}_{3}$ and $0.01 \mathrm{M} \mathrm{HNO}_{3}$, respectively. The solvent extraction experiments were performed at ambient temperature $\left(21 \pm 2{ }^{\circ} \mathrm{C}\right)$.

\subsection{Gas Chromatographic Analysis}

Samples of TRUEX solvent irradiated in contact with nitric acid were analyzed by gas chromatography with flame ionization detection (GC-FID) for the presence of potential degradation products as well as tri$n$-butylphosphate (TBP).

Due to the acidic functional groups of the dibutylphosphoric acid (HDBP) and monobutylphosphoric acid $\left(\mathrm{H}_{2} \mathrm{MBP}\right)$ degradation products of TBP , an aliquot of each TRUEX solvent sample was diluted 100 fold with hexane and derivatized with $300 \mu \mathrm{L}$ of a $\sim 0.3 \mathrm{~mol} / \mathrm{L}$ solution of diazomethane ${ }^{7}$ in diethyl ether prior to analysis. This produced the methyl ester of the phosphoric acid functional groups in the target compounds. Due to the large concentration of TBP relative to HDBP or $\mathrm{H}_{2} \mathrm{MBP}$ present in the samples, for TBP analyses samples were diluted 10,000 fold with hexane prior to analysis. The samples were analyzed along with appropriate calibration and quality assurance samples for TBP and the derivatized potential degradation products via GC-FID. Each sample was prepared following the same derivatization and preparation technique as necessary.

The gas chromatography analyses were performed on a Thermo Scientific Trace ULTRA gas chromatograph. The chromatograms were processed using Thermo Scientific Xcalibur software. The chromatographic separations were carried out utilizing a Thermo Scientific TG-35MS capillary column ( $30 \mathrm{~m} \times 0.25 \mathrm{~mm}$ ID $\times 0.25 \mu \mathrm{m}$ film). Analytical conditions were set at $2.0 \mathrm{~mL} / \mathrm{min}$ constant flow with helium as the carrier gas, with an $80 \mathrm{~mL} / \mathrm{min}$ split flow. Oven operating conditions started with a $2 \mathrm{~min}$ hold at $70^{\circ} \mathrm{C}$, followed by a ramp at $20^{\circ} \mathrm{C} / \mathrm{min}$ to $240{ }^{\circ} \mathrm{C}$ then $40^{\circ} \mathrm{C} / \mathrm{min}$ to $280^{\circ} \mathrm{C}$, finished with an 8.25 min hold at $280^{\circ} \mathrm{C}$. A Thermo AS3000 auto sampler (utilizing a $5 \mathrm{sec}$ pre-injection dwell time, performed a $1 \mu \mathrm{L}$ hot injection with the inlet set at $250^{\circ} \mathrm{C}$ ) is used for all injections. The FID is held at $250^{\circ} \mathrm{C}$. The fuel gas for the FID is a mixture of air and hydrogen. The air flow is set at $350 \mathrm{~mL} / \mathrm{min}$ and the hydrogen at $35 \mathrm{~mL} / \mathrm{min}$. Nitrogen is used as a makeup gas at $30 \mathrm{~mL} / \mathrm{min}$.

\subsection{Ion Chromatographic Analysis}

A Dionex (Sunnyvale, CA, USA) ICS-5000 ion chromatograph equipped with an eluent generator, an autosampler, dual quaternary gradient pumps with degas, conductivity detector, and an anion self- 
regenerating suppressor (ASRS) was used for all IC experiments. All columns and other consumables were obtained from Dionex. An IonPac AG5A analytical column (4mm x 150mm) and IonPac AG5A guard column ( $4 \mathrm{~mm} \times 35 \mathrm{~mm}$ ), a CR-ATC continuously regenerated anion trap column, and an ASRS 300 anion suppressor were used. The flow rate was $1.0 \mathrm{~mL} / \mathrm{min}$. The eluant generator was an EluGen II $\mathrm{KOH}$ cartridge. Deionized (DI) water generated by a water purification system (Barnstead E-Pure, ThermoScientific) was used for the preparation of all eluents, standards, and samples.

The procedure used for the IC analysis of the aqueous and organic samples is based upon the work of Dodi and Verda. ${ }^{8}$ Due to the varying concentration of the degradation species present in the irradiated samples, an aliquot of each aqueous sample was diluted between 20 and 100 fold with DI water. The samples were analyzed along with appropriate calibration and quality assurance samples. The gradient elution protocol used for the IC analyses is given in Table 1.

Table 1. Gradient elution protocol used for IC analysis.

\begin{tabular}{c|c} 
Time, min & {$\left[\mathrm{OH}^{-}\right], \mathrm{mM}$} \\
\hline $0.0-4.0$ & 3.0 \\
$4.0-14.0$ & 10.0 \\
$14.0-20.5$ & 23.0 \\
$20.5-24.0$ & 55.0 \\
$24.0-30.0$ & 3.0
\end{tabular}

\subsection{High Performance Liquid Chromatographic Analysis}

High Performance Liquid Chromatography (HPLC) was used to quantify CMPO in the irradiated TRUEX solvent. The HPLC analyses were performed using Shimadzu (Kyoto, Japan) LC-10 AD VP pumps, a CTO-10 AC VP column oven, a SIL-HTc autosampler, a SPD-M10A VP photo-diode array detector, and Class VP software. All experiments conducted on the Shimadzu HPLC were carried out isocratically using a mixture of $30 \mathrm{mM}$ phosphate buffer, $\mathrm{pH} \sim 2.6$, and 2-propanol with 3.6 \% 1-octanol in 60/40 ratio. The chromatographic separation was achieved with a $\mathrm{C}_{18}$ reverse-phase $\left(\mathrm{RP}-\mathrm{C}_{18}\right)$ column (Supelco, $25 \mathrm{~cm} \times 4.6 \mathrm{~mm}, 5 \mu \mathrm{m}$ ) with a flow rate of $1 \mathrm{~mL} / \mathrm{min}$. The column temperature was maintained at $50^{\circ} \mathrm{C}$. The CMPO concentration was determined at a wavelength of $220 \mathrm{~nm}$.

Stock standard solutions of CMPO $(0.1 \mathrm{M})$ were prepared in dodecane and were diluted in 2-propanol to prepare different CMPO concentrations ranging from $0.05 \mathrm{mM}$ to $1 \mathrm{mM}$. These were used to build calibration curves using the HPLC-UV analytical technique. An internal standard, triphenylphosphate (TPP) was added at a concentration of $1 \mathrm{mM}$ prior to analyses of samples, which improved the measurement accuracy by correcting for detector response variations

\section{Results - Hydrolysis and Radiolysis Experiments in Test Loop}

Mixtures of TRUEX solvent and 4.4 M nitric acid were irradiated using the INL test loop and ${ }^{60} \mathrm{Co}-\gamma$ irradiation from the Nordion GammaCell 200E (Ottawa, Canada) irradiator with a current sample chamber center-line dose rate of $6.5 \mathrm{kGy} / \mathrm{hr}$. The effective absorbed gamma dose rate of $3.5 \mathrm{kGy} / \mathrm{hr}$ delivered to samples in the test loop was based upon decay-corrected Fricke dosimetry, ${ }^{9}$ the photobleaching of a methyl red solution due to gamma irradiation, ${ }^{10}$ and the duration of each irradiation. 
The variation in the aqueous and organic phase nitric acid concentration was determined as a function of absorbed dose. The acid concentrations were determined by titration with standard base to a phenolphthalein end point. The concentration of nitric acid present in the aqueous phase is plotted as a function of absorbed dose in Figure 3. The concentration of acid present in the aqueous phase at zero absorbed dose is only $\sim 3.5 \mathrm{M} \mathrm{HNO}_{3}$ due to the extraction of nitric acid into the TRUEX solvent. The observed increase in acid concentration as a function of absorbed dose is due to the sampling protocol used during the radiolysis study. After removal of a volume of aqueous and organic phases from the Test Loop for analysis, a volume of $4.4 \mathrm{M} \mathrm{HNO}_{3}$ equal to the total solution sample volume was added back to the loop. This acid addition was performed to maintain a constant solution volume in the Test Loop.

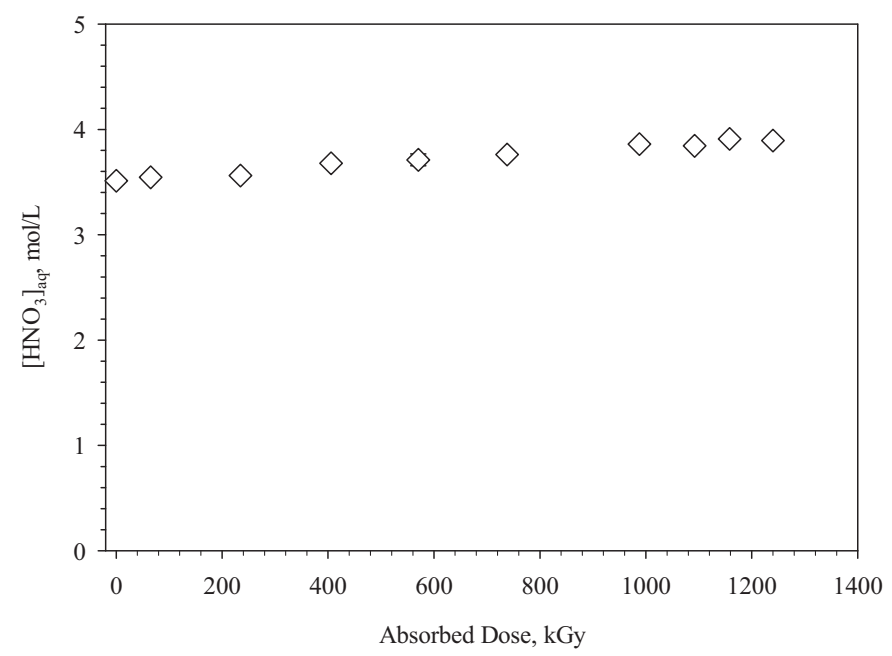

Figure 3. Concentration of $\mathrm{HNO}_{3}$ present in the aqueous phase versus absorbed dose for nitric acid in contact with TRUEX solvent. Diamond data markers: determined acid concentration.

The concentration of nitric acid present in the organic phase is plotted as a function of absorbed dose in Figure 4. Due to the limited volume of irradiated organic available, these titrations were performed using relatively small $(<20 \mu \mathrm{L})$ sample volumes. The organic sample was suspended in de-ionized water in order to partition the acid to the aqueous phase and the resultant aqueous phase was titrated to determine the concentration of acid in the organic sample. An approximately $20 \%$ decrease in the concentration of nitric acid dissolved in the organic phase is observed over the course of the radiolysis experiment. The decrease in the concentration of nitric acid in the organic phase as a function of absorbed dose is consistent with the destruction of TBP and CMPO due to radiolytic degradation and the subsequent decrease in the extent of acid loading in the organic phase. It should be noted that the titration methodology used to determine the acid concentration in the organic phase would not titrate the acidic proton of dibutylphosphoric acid. Dibutylphosphoric acid is a major degradation product of TBP formed during radiolysis and the relatively high partitioning coefficient, $K_{D}=143$, (vide infra) would limit the partitioning of the dibutylphosphoric acid to the aqueous phase. 


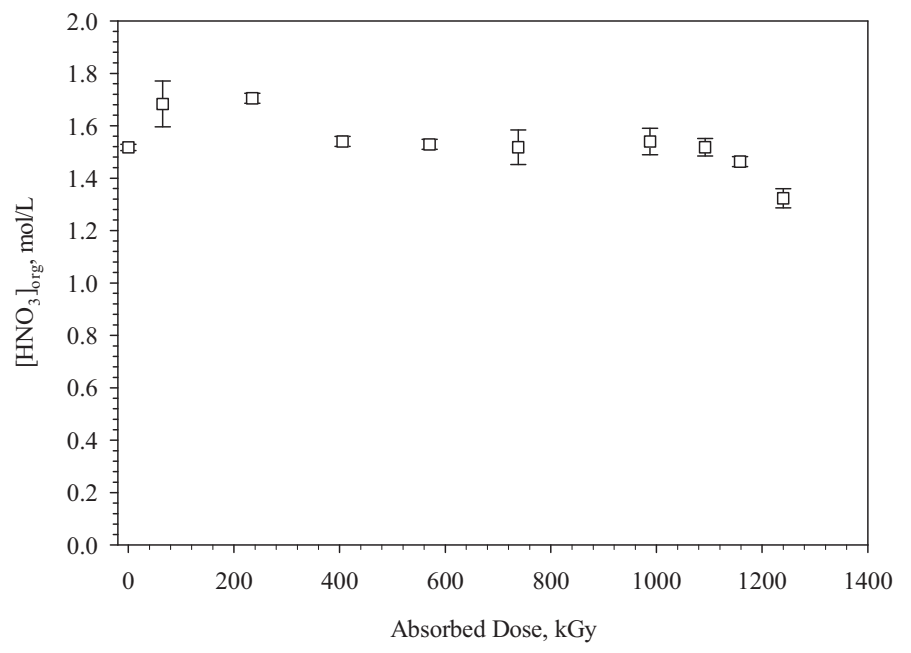

Figure 4. Concentration of $\mathrm{HNO}_{3}$ present in the organic phase versus absorbed dose for TRUEX solvent in contact with nitric acid.

\subsection{Solvent Extraction Experiments}

\subsubsection{Solvent Hydrolysis}

The results of a TRUEX solvent hydrolysis study performed in the INL Test Loop have been reported previously ${ }^{11}$ and are only summarized here. A sample of TRUEX solvent and 4.4 $\mathrm{M} \mathrm{HNO}_{3}$ was placed in the Test Loop and allowed to circulate while being well-mixed for several days. The mixed phases were not irradiated using the gamma irradiator. In order to accelerate any hydrolytic degradation processes, the temperature of the solution circulating in the test loop was maintained at $50{ }^{\circ} \mathrm{C}$ using the external temperature-controlled water bath. The measured americium and europium distribution ratios for the extraction and scrub sections of the flowsheet exhibited no statistically significant variation with contact time. The strip distribution ratios were all less than 0.001 . No statistically significant variation in either the wash or rinse distribution ratios was observed. Based upon these observations, acid hydrolysis was not considered to be a process with regards to the degradation of TRUEX solvent.

\subsubsection{Solvent Radiolysis}

A sample of TRUEX solvent and 4.4 $\mathrm{M} \mathrm{HNO}_{3}$ was placed in the test loop and allowed to circulate while being well-mixed. The mixed phases were irradiated in the gamma irradiator until an absorbed gamma dose of approximately $1200 \mathrm{kGy}$ was achieved. Samples of the aqueous and organic phases were removed during the course of the irradiation. A volume of fresh nitric acid corresponding to the total volume of aqueous and organic removed at each sampling point was added to the test loop to maintain a constant volume of solution in the loop. The samples of aqueous and organic phases taken from the loop were used to perform an extraction/scrub/strip batch distribution ratio determination for americium and europium as a function of absorbed dose. 
The americium and europium extraction, scrub, and strip distribution ratios determined as a function of absorbed dose are presented in Tables 2 and 3, respectively. The extraction and strip distribution ratios are also plotted in Figures 5 and 6, respectively. Since the scrub distribution ratios generally followed the trends observed in the case of the extraction distribution ratios, a plot of the americium and europium scrub distribution ratios versus absorbed dose is not presented here. The determined Am and Eu extraction and scrub distribution ratios decline steadily as the absorbed gamma dose increases. The determined Am and Eu stripping distribution ratios exhibit a steady increase with absorbed dose. The slopes of the plots of the extraction, scrub, and strip distribution ratios are similar for both Am and Eu. This indicates that both metals are bound by the TRUEX extractants in a similar manner.

These results indicate that combination of radiolytic destruction of the TRUEX solvent components and the generation of radiolytic degradation products adversely impacts the efficacy of the TRUEX solvent. Previous experiments performed on the TRUEX process solvent in the INL Radiolysis Test Loop ${ }^{11}$ demonstrated that a caustic wash was effective at removing the majority of radiolytic degradation products formed during radiolysis. In addition, the previous results demonstrated that the aqueous complexant, diethylenetriaminepentaacetic acid (DTPA), employed in the stripping section of a typical TRUEX process flowsheet was capable of efficient stripping even after an absorbed gamma dose of 860 kGy.

Table 2. Am distribution ratios for TRUEX solvent irradiated in contact with $4.4 \mathrm{M} \mathrm{HNO}_{3}$ as a function of absorbed dose.

\begin{tabular}{|c|c|c|c|}
\hline Absorbed Dose, kGy & \multicolumn{3}{|c|}{ Am Distribution Ratios } \\
\hline & Extraction & Scrub & Strip \\
\hline 0.0 & $35.3 \pm 1.3$ & $37.3 \pm 3.6$ & $0.67 \pm 0.15$ \\
\hline 64.9 & $32.8 \pm 0.2$ & $38.1 \pm 0.4$ & $0.74 \pm 0.01$ \\
\hline 234.5 & $31.7 \pm 1.2$ & $30.4 \pm 1.4$ & $0.91 \pm 0.06$ \\
\hline 405.7 & $26.9 \pm 0.6$ & $30.1 \pm 3.5$ & $0.92 \pm 0.09$ \\
\hline 570.3 & $29.4 \pm 2.5$ & $26.2 \pm 0.8$ & $1.14 \pm 0.07$ \\
\hline 738.2 & $24.7 \pm 0.8$ & $25.7 \pm 0.3$ & $1.46 \pm 0.07$ \\
\hline 987.7 & $24.5 \pm 1.5$ & $25.5 \pm 0.3$ & $1.88 \pm 0.08$ \\
\hline 1092 & $22.3 \pm 0.6$ & $24.3 \pm 2.1$ & $2.15 \pm 0.11$ \\
\hline 1158 & $23.2 \pm 1.6$ & $25.4 \pm 0.7$ & $2.13 \pm 0.07$ \\
\hline 1240 & $21.3 \pm 0.1$ & $21.5 \pm 1.5$ & $1.94 \pm 0.07$ \\
\hline
\end{tabular}


Table 3. Eu distribution ratios for TRUEX solvent irradiated in contact with $4.4 \mathrm{M} \mathrm{HNO}_{3}$ as a function of absorbed dose.

\begin{tabular}{|c|c|c|c|}
\hline Absorbed Dose, kGy & \multicolumn{3}{|c|}{ Eu Distribution Ratios } \\
\hline & Extraction & Scrub & Strip \\
\hline 0.0 & $25.4 \pm 0.8$ & $23.2 \pm 0.3$ & $0.48 \pm 0.05$ \\
\hline 64.9 & $24.5 \pm 0.1$ & $22.7 \pm 0.3$ & $0.49 \pm 0.02$ \\
\hline 234.5 & $22.3 \pm 0.9$ & $19.8 \pm 0.9$ & $0.56 \pm 0.03$ \\
\hline 405.7 & $19.5 \pm 0.9$ & $18.7 \pm 0.9$ & $0.62 \pm 0.03$ \\
\hline 570.3 & $19.5 \pm 0.5$ & $16.3 \pm 0.5$ & $0.81 \pm 0.01$ \\
\hline 738.2 & $19.3 \pm 0.6$ & $16.9 \pm 0.9$ & $1.02 \pm 0.03$ \\
\hline 987.7 & $17.2 \pm 0.5$ & $15.0 \pm 0.3$ & $1.45 \pm 0.02$ \\
\hline 1092 & $15.9 \pm 0.2$ & $14.5 \pm 0.2$ & $1.59 \pm 0.09$ \\
\hline 1158 & $17.1 \pm 0.4$ & $16.2 \pm 0.4$ & $1.67 \pm 0.01$ \\
\hline 1240 & $14.9 \pm 0.2$ & $13.1 \pm 0.3$ & $1.55 \pm 0.03$ \\
\hline
\end{tabular}

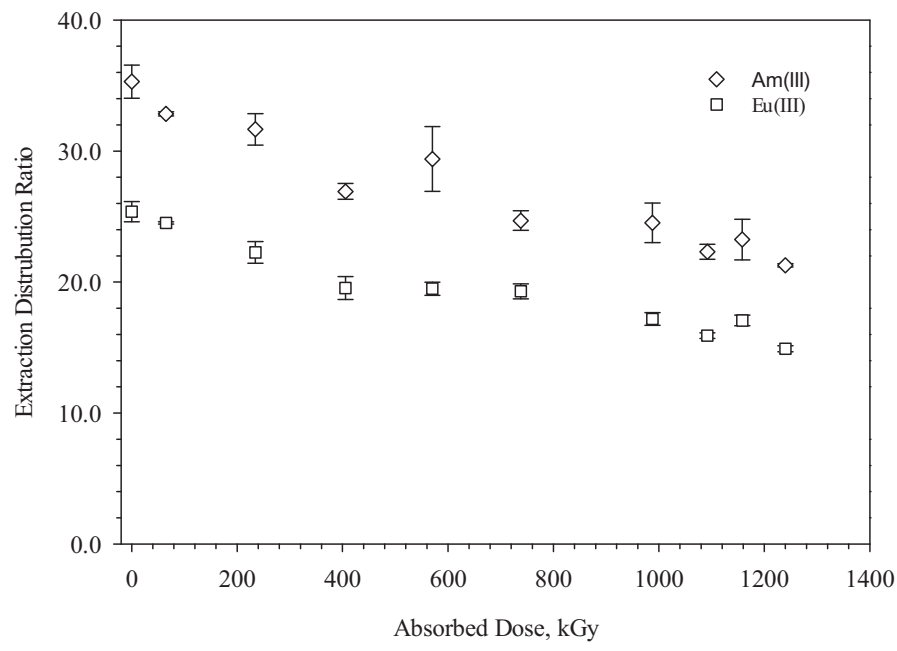

Figure 5. Measured extraction distribution ratios of Am (open diamonds) and Eu (open squares) for TRUEX solvent irradiated in the INL TEST loop in contact with $4.4 \mathrm{M} \mathrm{HNO}_{3}$ as a function of absorbed dose. 


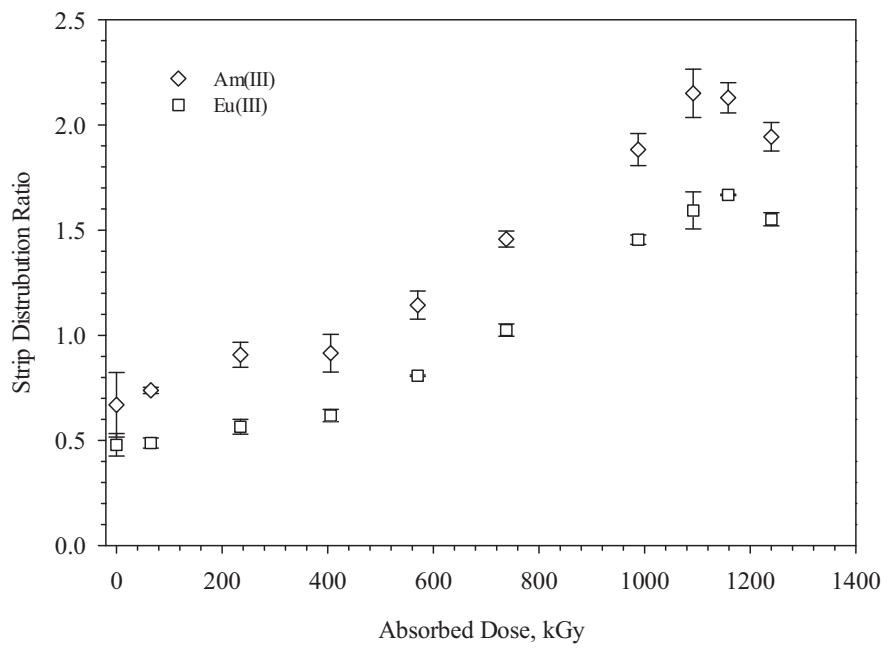

Figure 6. Measured stripping distribution ratios of $\mathrm{Am}$ (open diamonds) and $\mathrm{Eu}$ (open squares) for TRUEX solvent irradiated in the INL TEST loop in contact with $4.4 \mathrm{M} \mathrm{HNO}_{3}$ as a function of absorbed dose.

\subsection{Gas Chromatographic Analysis}

\subsubsection{Solvent Hydrolysis}

The results of a TRUEX solvent hydrolysis have been reported previously ${ }^{11}$ and are only summarized here. The samples from the TRUEX solvent hydrolysis test were also analyzed by gas chromatography with flame ionization detection (GC-FID) in order to determine the variation in the composition of the solvent due to hydrolytic degradation. The nominal composition of the TRUEX solvent is $1.4 \mathrm{M} \mathrm{TBP}+$ $0.2 \mathrm{M}$ CMPO dissolved in n-dodecane. No statistically significant variation of the concentration of TBP or CMPO over the course of the hydrolysis test is observed. A small, monotonic increase in the measured concentration of dibutylphosphoric acid (HDBP) was observed.

\subsubsection{Solvent Radiolysis}

The samples from the TRUEX solvent radiolysis test were analyzed by GC-FID in order to determine the variation in the composition of the solvent due to radiolytic degradation. The nominal composition of the TRUEX solvent is $1.4 \mathrm{M}$ TBP + 0.2 M CMPO dissolved in n-dodecane. A typical GC-FID chromatogram observed for the analysis of TBP is shown in Figure 7. In the chromatogram, n-dodecane and TBP elute at RT $=6.5 \mathrm{~min}$ and $10.4 \mathrm{~min}$, respectively. The peak at $\mathrm{RT}=8.5 \mathrm{~min}$ corresponds to 2 dodecanol which is used as an internal injection standard. A typical chromatogram observed for the analysis of $\mathrm{HDBP}$ and $\mathrm{H}_{2} \mathrm{MBP}$ is shown in Figure 8. Under the conditions employed for the analysis of acidic degradation products, the observed RT for $n$-dodecane and TBP are shifted to higher values due to the large amount of these species injected onto the GC column. The amount of 2-dodecanol injection standard used was adjusted to correspond more closely with the concentration of the target analyte. 


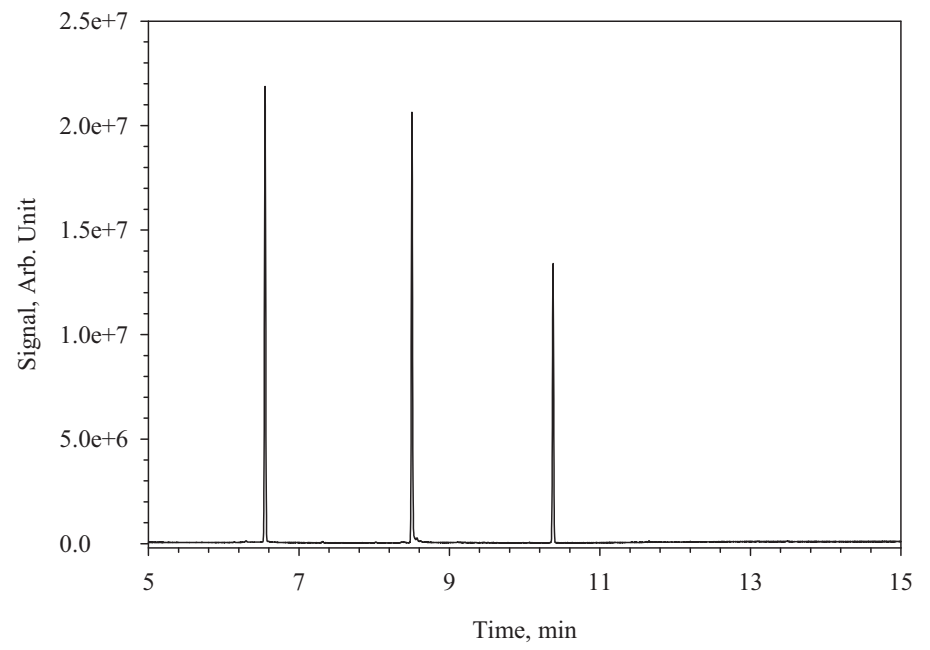

Figure 7. Typical GC-FID chromatogram for TBP analysis. Retention times: RT $=6.5$ min, n-dodecane; $\mathrm{RT}=8.5 \mathrm{~min}, 2$-dodecanol; $\mathrm{RT}=10.4 \mathrm{~min}$, TBP.

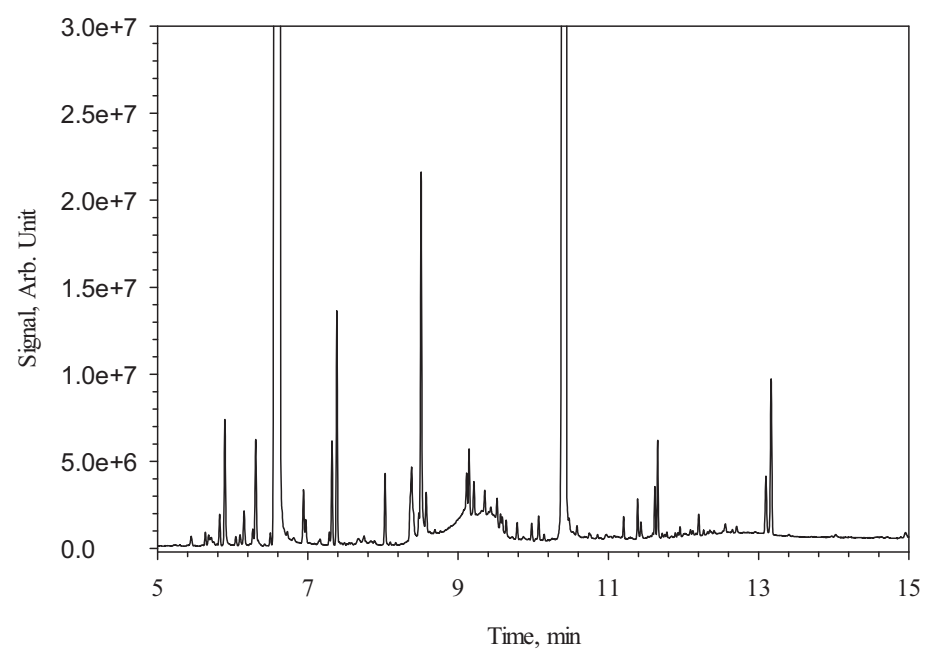

Figure 8. Typical GC-FID chromatogram for $\mathrm{HDBP}$ and $\mathrm{H}_{2} \mathrm{MBP}$ analysis. Retention times: $\mathrm{RT}=7.6$ min, methyl ester of $\mathrm{H}_{2} \mathrm{MBP}$; $\mathrm{RT}=9.2$ min, methyl ester of HDBP.

The measured concentrations of TBP and HDBP (the major acidic radiolytic degradation product of TBP) present in the TRUEX solvent as a function of absorbed dose are presented in Figure 9 and 10, respectively. Over the course of the irradiation, the determined concentration of TBP exhibits a statistically significant decrease of approximately $0.14 \mathrm{M}$, while the determined concentration of HDBP increased by approximately $0.13 \mathrm{M}$ over the course the radiolysis experiment. Over the same range of absorbed dose, the concentration of nitric acid dissolved in the organic phase decreased by approximately $0.2 \mathrm{M}$. 


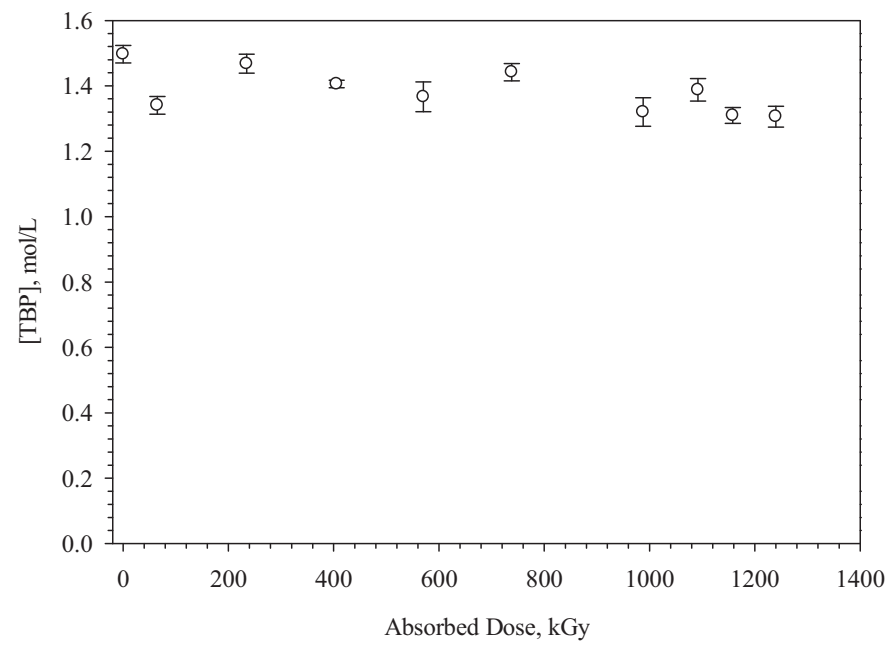

Figure 9. Concentration of TBP determined by GC-FID analysis present in the irradiated TRUEX solvent as a function of absorbed dose.

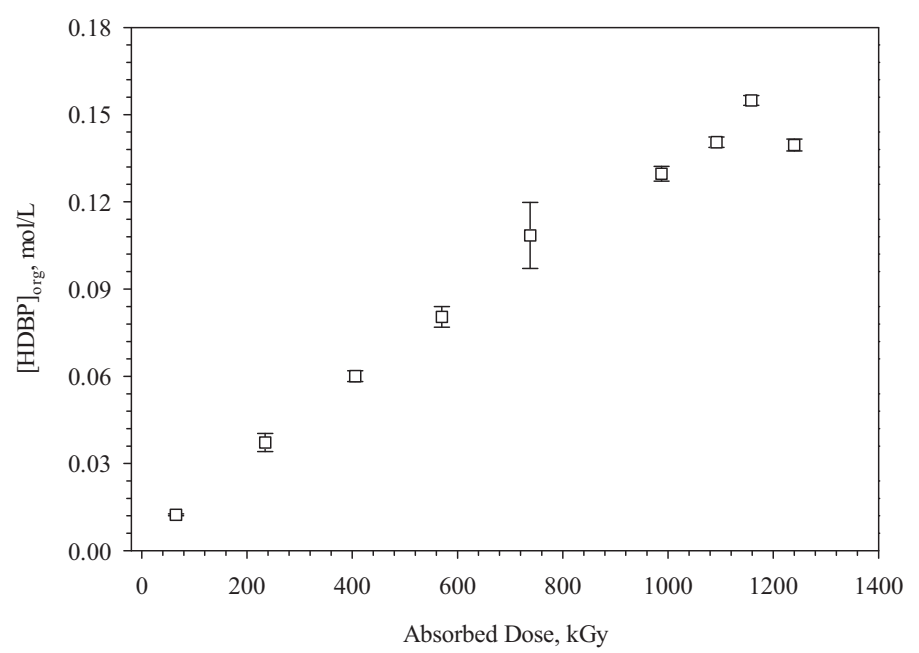

Figure 10. Concentration of HDBP determined by GC-FID analysis present in the irradiated TRUEX solvent as a function of absorbed dose.

\subsection{Ion Chromatographic Analysis}

The aqueous nitric acid phase was analyzed by anionic ion chromatography in order to determine the concentration of aqueous soluble radiolytic degradation products. A typical ion chromatograph for the analysis of a nitric acid phase with an absorbed dose of $785 \mathrm{kGy}$ is shown in Figure 11. It is clearly evident that the ion chromatograph is dominated by the nitrate anion, which elutes from approximately 5 $\min -10 \mathrm{~min}$. 


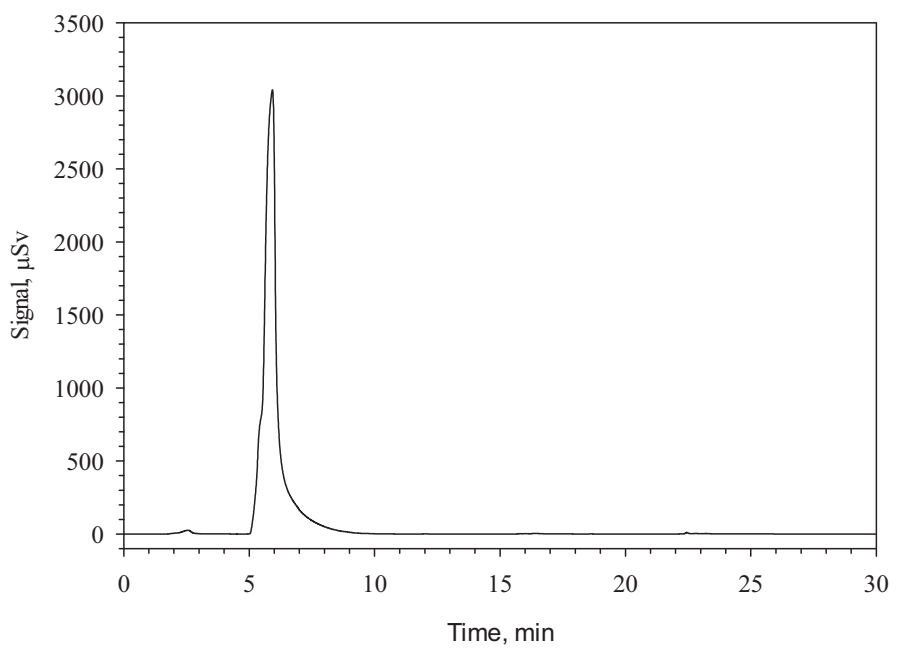

Figure 11. Ion chromatograph of aqueous nitric acid phase following irradiation to an absorbed dose of $785 \mathrm{kGy}$. Peak eluting between 5 - 10 min due to nitrate anion.

An expanded y-axis view of the chromatogram shown in Figure 11 is presented in Figure 12. The anions of HDBP and $\mathrm{H}_{2} \mathrm{MBP}$ are minor peaks in the chromatogram with $\mathrm{RT}=4 \mathrm{~min}$ and $12 \mathrm{~min}$, respectively. The phosphate anion elutes last with $\mathrm{RT}=22.5 \mathrm{~min}$. Two peaks corresponding to unknown degradation products are observed with $\mathrm{RT}=2.5 \mathrm{~min}$ and $15.5-17.5 \mathrm{~min}$, respectively.

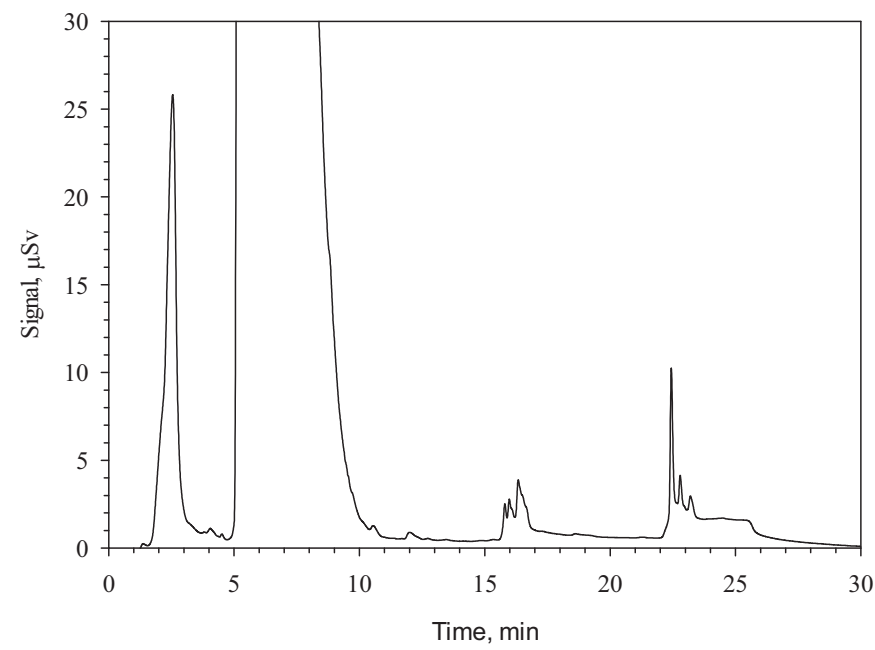

Figure 12. Ion chromatograph of aqueous nitric acid phase following irradiation to an absorbed dose of $785 \mathrm{kGy}$ - expanded y-axis. Retention times: $\mathrm{RT}=2.5 \mathrm{~min}$, unknown; RT $=4 \mathrm{~min}$, anion of HDBP; RT $=5-10 \mathrm{~min}$, nitrate anion; $\mathrm{RT}=12 \mathrm{~min}$, anion of $\mathrm{H}_{2} \mathrm{MBP} ; \mathrm{RT}=15.5-17.5 \mathrm{~min}$, unknown; $\mathrm{RT}=22.5$ min, phosphate anion.

The concentrations of anion of HDBP, anion of $\mathrm{H}_{2} \mathrm{MBP}$, and phosphate anion present in the irradiated aqueous phase as a function of absorbed dose are plotted in Figures 13 - 15, respectively. 
The dependence of $[\mathrm{HDBP}]_{\mathrm{aq}}$ on absorbed dose, see Figure 13, demonstrates that a small fraction of the HDBP produced by the radiolysis of TBP is soluble in the aqueous nitric acid phase. Approximately $0.001 \mathrm{M}$ HDBP is found in the aqueous nitric phase following irradiation of the TRUEX-nitric acid mixture. The concentration of HDBP determined in the aqueous and organic phase as a function of absorbed dose can be used to calculate a value of the partition coefficient for HDBP, $\mathrm{K}_{\mathrm{D}}=$ $[\mathrm{HDBP}]_{\text {org }} /[\mathrm{HDBP}]_{\mathrm{aq}}$. The partition coefficient for HDBP determined in this work is $\mathrm{K}_{\mathrm{D}}(\mathrm{HDBP})=140 \pm$ 33.

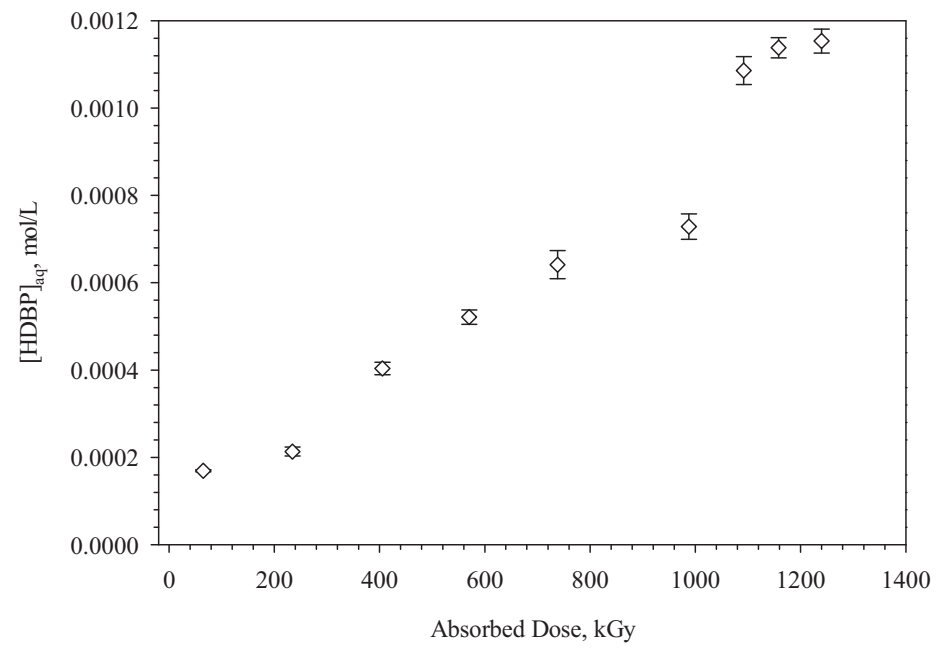

Figure 13. Concentration of HDBP determined by IC analysis present in the irradiated $\mathrm{HNO}_{3}$ as a function of absorbed dose.

A relatively minor amount of $\mathrm{H}_{2} \mathrm{MBP}$ is detected (see Figure 14) in the aqueous samples taken at greater than approximately $1000 \mathrm{kGy}$ absorbed dose. The small concentrations of $\mathrm{H}_{2} \mathrm{MBP}$ are consistent with the requirement that HDBP must first be produced via radiolysis of TBP and undergo subsequent radiolysis to form $\mathrm{H}_{2} \mathrm{MBP}$. The formation of $\mathrm{H}_{2} \mathrm{MBP}$ directly from TBP via radiolysis is not a likely degradation pathway.

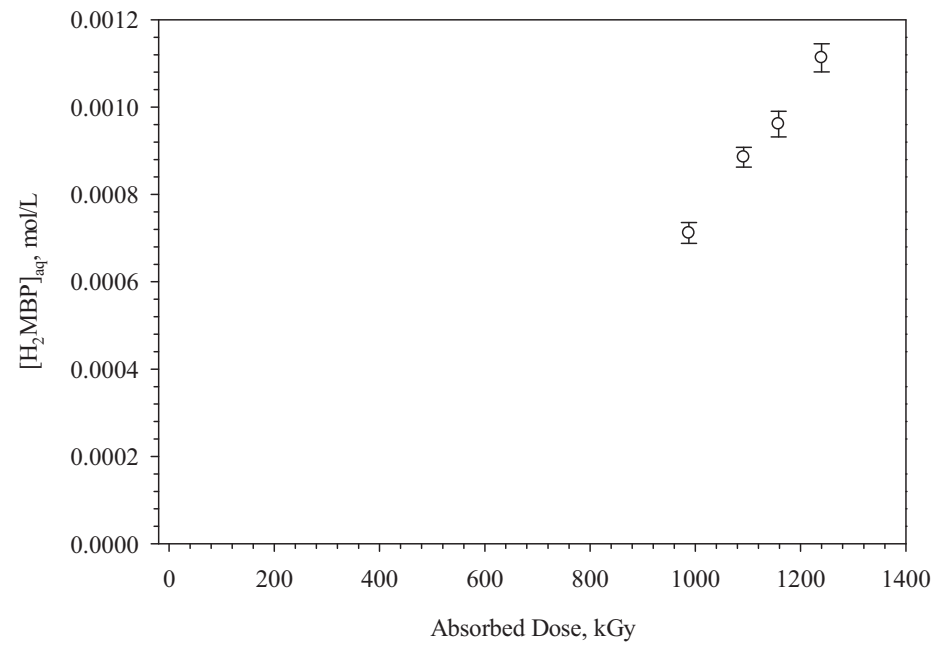

Figure 14. Concentration of $\mathrm{H}_{2} \mathrm{MBP}$ determined by $\mathrm{IC}$ analysis present in the irradiated $\mathrm{HNO}_{3}$ as a function of absorbed dose. 
As was observed in the case of $\mathrm{H}_{2} \mathrm{MBP}$, a relatively minor amount of phosphate anion is detected (see Figure 15) in the aqueous samples taken from the TRUEX radiolysis experiment. Curiously, a larger amount of phosphate anion is detected relative to the detected concentration of $\mathrm{H}_{2} \mathrm{MBP}$.

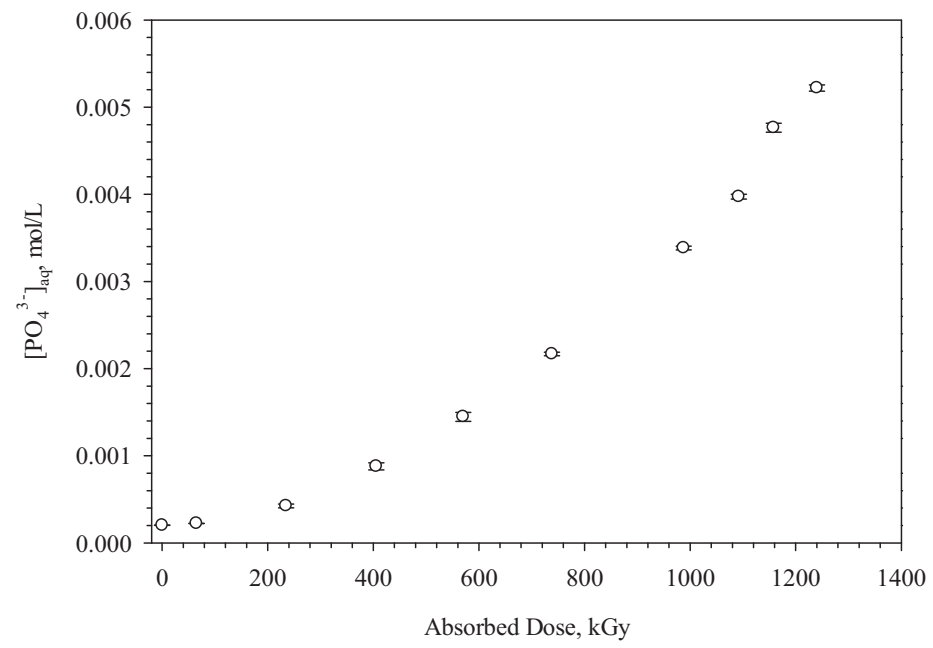

Figure 15. Concentration of phosphate anion determined by IC analysis present in the irradiated $\mathrm{HNO}_{3}$ as a function of absorbed dose.

The determined peak areas for two unknown compounds detected in the irradiated nitric acid phase are plotted as a function of absorbed dose in Figure 16. Based upon the observed peak areas, the peak at RT $=16.8 \mathrm{~min}$ (square data markers in Figure 16) likely corresponds to a minor degradation product. The significantly larger peak areas observed for the $\mathrm{RT}=2.5 \mathrm{~min}$ peak (diamond data markers in Figure 16) suggest that this peak corresponds to either a major degradation product or several degradation products with similar charge. In addition, the decrease in the observed peak area at absorbed doses greater than $1000 \mathrm{kGy}$ indicate that this degradation product undergoes further radiolysis at high absorbed doses.

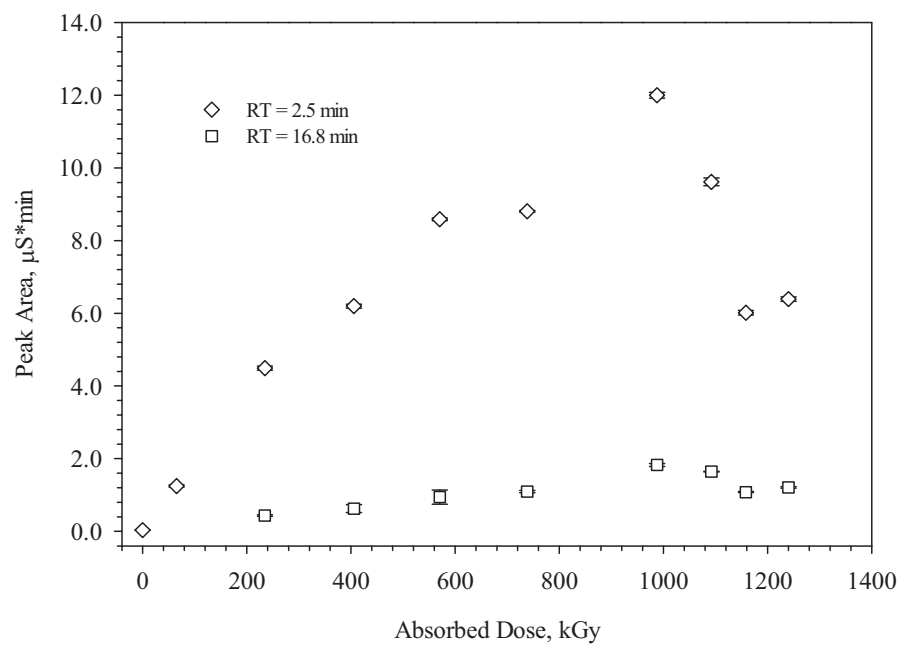

Figure 16. Plot of peak area versus absorbed dose for two unidentified products of TRUEX radiolysis determined by IC analysis of the irradiated 4.4 $\mathrm{M} \mathrm{HNO}_{3}$. Retention times: $2.5 \mathrm{~min}$ (diamonds) and 16.8 min (squares). 


\subsection{High Performance Liquid Chromatographic Analysis}

The organic phase from the TRUEX radiolysis experiment was analyzed by HPLC in order to determine the concentration of CMPO and presence of other organic soluble radiolytic degradation products. A typical HPLC chromatogram is shown in Figure 17. The peak corresponding to CMPO has a retention time of 15.0 min. The peak observed at RT $=7.5$ is due to triphenyl phosphate which is utilized as an internal standard in the HPLC analyses.

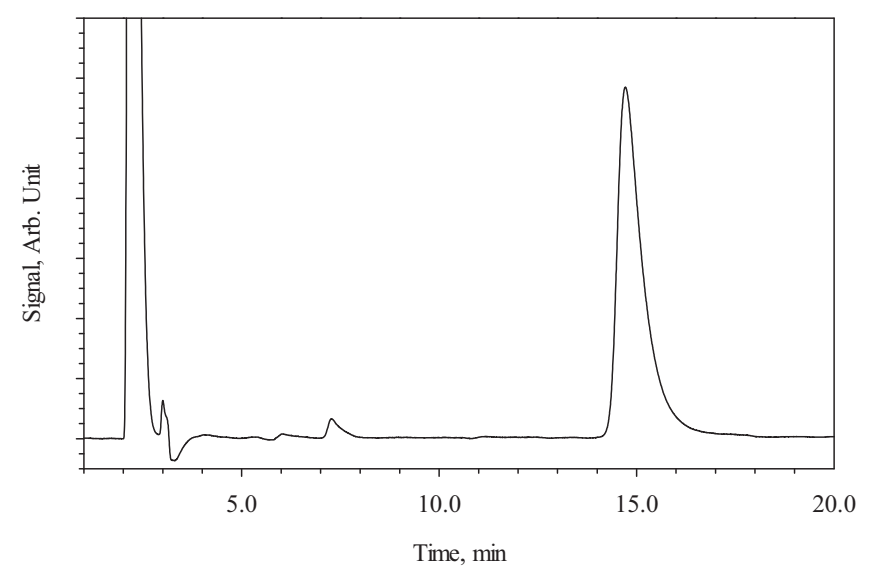

Figure 17. Typical HPLC chromatogram for the analysis of TRUEX solvent. Retention times: RT $=7.5$ min, triphenyl phosphate (used as internal standard); RT = $15.0 \mathrm{~min}, \mathrm{CMPO}$.

The concentration of CMPO as a function of absorbed dose is presented in Figure 18. The concentration of CMPO decreases with increasing absorbed dose up to approximately $800 \mathrm{kGy}$. At absorbed doses greater than approximately $1000 \mathrm{kGy}$, no statistically significant variation in the analytically determined concentration of CMPO is observed.

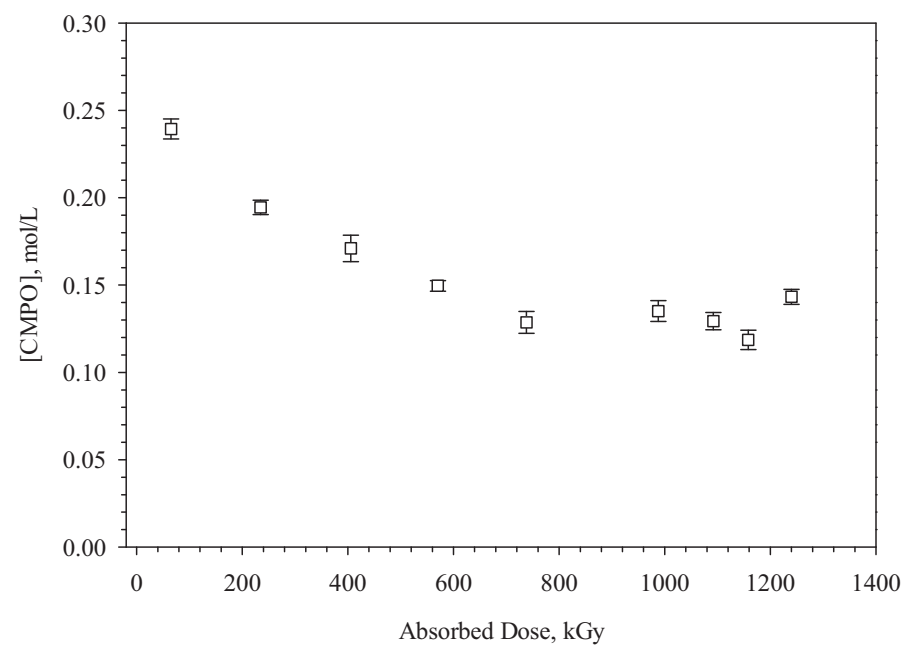

Figure 18. Concentration of CMPO determined by HPLC analysis present in the irradiated TRUEX solvent as a function of absorbed dose.

The change in peak areas of three unknown radiolytic degradation products which were detected by the HPLC analysis as a function of absorbed dose are presented in Figure 19. 


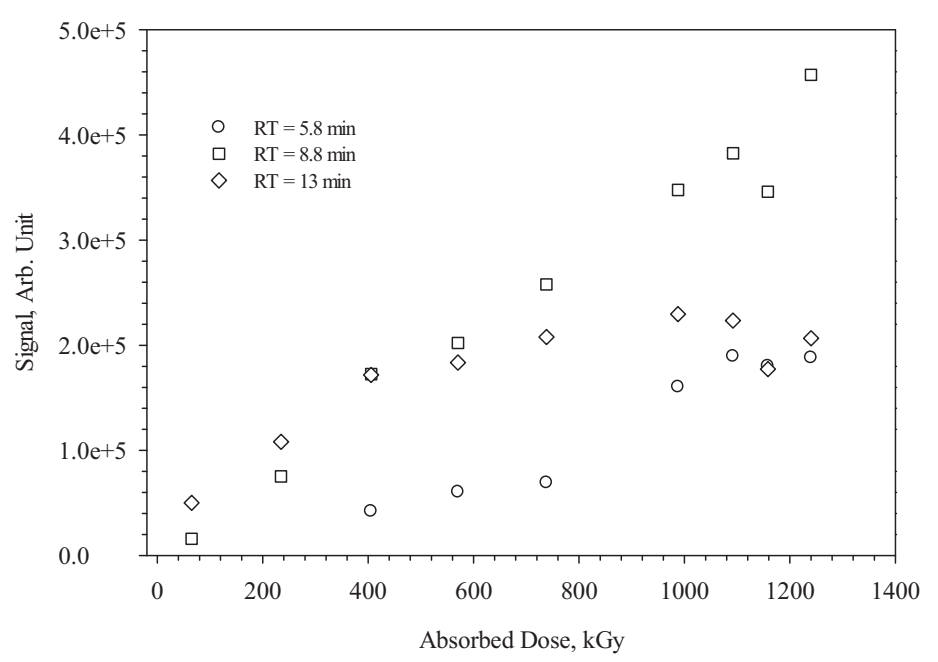

Figure 19. Plot of peak area versus absorbed dose for three unidentified products of TRUEX radiolysis determined by HPLC analysis of the irradiated TRUEX solvent. Retention times: 5.8 min (circles), 8.8 min (squares) and 13 min (diamonds).

The peak area corresponding to the unknown TRUEX radiolytic degradation product with a retention time, RT $=8.8 \mathrm{~min}$, (open squares in Figure 17) increases with increasing absorbed dose. Similar to what is observed in the case of the CMPO concentration versus absorbed dose determined by HPLC, the peak areas of the remaining unknown organic soluble TRUEX degradation products, RT $=5.8$ and 13 min, (open circles and open diamonds in Figure 19, respectively) increase with increasing dose but show no statistically significant variation above approximately $1000 \mathrm{kGy}$ absorbed dose.

\section{Discussion}

The INL radiolysis and hydrolysis Test Loop has been used to evaluate the effects of hydrolytic and radiolytic degradation upon the efficacy of the TRUEX flowsheet for the recovery of trivalent actinides and lanthanides from acidic solution. The results demonstrate that solvent hydrolysis does not adversely impact the performance of the process flowsheet. However, hydrolysis does likely account for the small amount of HDBP present in the un-irradiated solvent.

The nominal composition of the TRUEX solvent used in this study is $0.2 \mathrm{M}$ CMPO and 1.4 M TBP dissolved in n-dodecane. Gamma irradiation of a mixture of TRUEX process solvent and nitric acid in the test loop does adversely impact flowsheet performance as measured by the decreasing americium and europium distribution ratios in the extraction section as accumulated dose increases. The observed increase in americium and europium stripping distribution ratio with increasing absorbed dose indicates the radiolytic production of organic soluble degradation compounds. These organic soluble degradation products inhibit stripping of the irradiated TRUEX solvent with dilute nitric acid. Use of an aqueous complexant, i.e. DTPA, in the aqueous strip solution easily overcomes the decreased stripping performance.

The efficiency of the conversion of absorbed radiation energy (gamma rays) into chemical products (radiolytic degradation products) is here defined as the $G$-value, in units of $\mu \mathrm{mol} \cdot \mathrm{L}^{-1} \cdot \mathrm{Gy}^{-1}$. The test loop was previously used to study the radiolysis of TBP/n-dodecane solvent mixed with $0.9-3.0 \mathrm{M} \mathrm{HNO}_{3}$. The $G$-value for the radiolytic degradation of TBP determined in that work was $0.12 \mu \mathrm{mol} \cdot \mathrm{L}^{-1} \cdot \mathrm{Gy}^{-1}{ }^{12}$ The 
$G$-values presented below are summarized in Table 4. The concentration of TBP determined in this work as a function of absorbed dose is plotted in Figure 20 as $\mu \mathrm{mol} / \mathrm{L}$ TBP versus Gy. The slope of the linear regression of the data presented in Figure 20 corresponds to the $G$-value for the radiolytic destruction of TBP, $-G_{\mathrm{TBP}}=-0.114 \pm 0.031 \mu \mathrm{mol} \cdot \mathrm{L}^{-1} \cdot \mathrm{Gy}^{-1}$. The $G$-value for the radiolytic degradation of TBP agrees well with previous determinations conducted at the INL.

Table 4. Summary of $G$-values determined in this work.

\begin{tabular}{|c|c|c|}
\hline Species & $G$-value, $\mu \mathrm{mol} \cdot \mathrm{L}^{-1} \cdot \mathrm{Gy}^{-1}$ & $\mathrm{R}^{2}$ of linear regression \\
\hline TBP & $-0.115 \pm 0.031$ & 0.62 \\
\hline CMPO & $-0.159 \pm 0.016$ & 0.97 \\
\hline $\mathrm{HDBP}_{\text {tot }}$ & $0.118 \pm 0.007$ & 0.97 \\
\hline $\mathrm{H}_{2} \mathrm{MBP}$ & $1.56 \times 10^{-3} \pm 8.06 \times 10^{-5}$ & 0.99 \\
\hline Phosphate & $5.66 \times 10^{-3} \pm 3.94 \times 10^{-4}$ & 0.98 \\
\hline
\end{tabular}

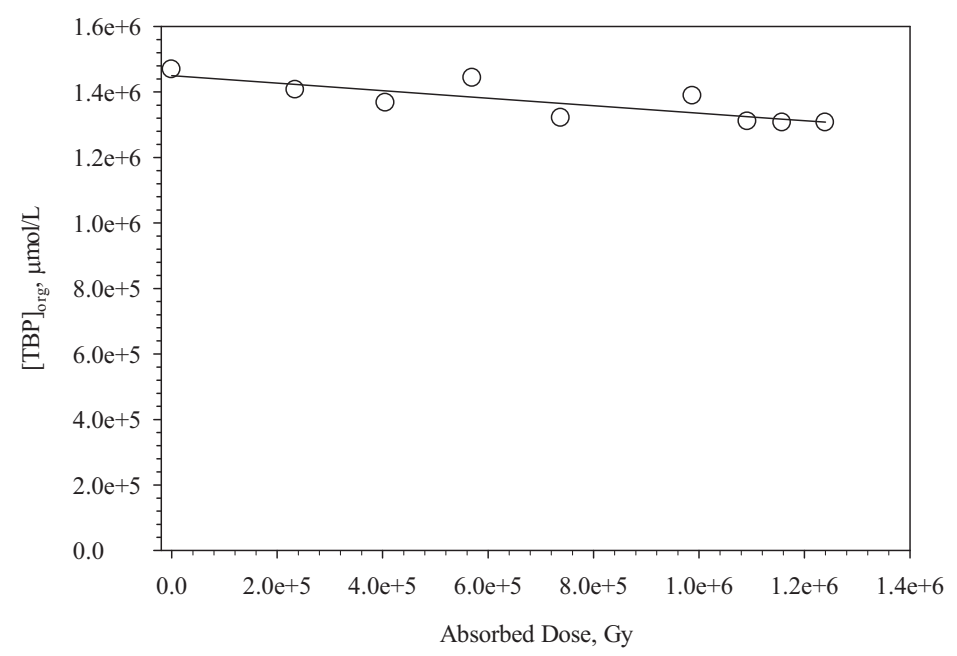

Figure 20. Plot of the concentration of TBP versus absorbed dose for TRUEX solvent irradiated in contact with 4.4 $\mathrm{M} \mathrm{HNO}_{3}$. The slope of the linear best-fit line corresponds to the $G$-value for the decomposition of TBP. The determined $G$-value for the destruction of TBP is $-G_{\mathrm{TBP}}=-0.114 \pm 0.031$ $\mu \mathrm{mol} \cdot \mathrm{L}^{-1} \cdot \mathrm{Gy}^{-1} \cdot\left(\mathrm{R}^{2}=0.62\right)$

The concentration of CMPO determined in this work as a function of absorbed dose is plotted in Figure 21 as $\mu \mathrm{mol} / \mathrm{L} \mathrm{CMPO}$ versus Gy. In order to determine a $G$-value for the destruction of CMPO, the linear portion of the CMPO concentration versus absorbed dose plot is linearly regressed. The slope of the bestfit linear regression fit to the linear portion of the data plotted in Figure 21 is, $-G_{\mathrm{CMPO}}=-0.159 \pm 0.016$ $\mu \mathrm{mol} \cdot \mathrm{L}^{-1} \cdot \mathrm{Gy}^{-1}$. The lack of a statistically significant variation in the concentration of CMPO at absorbed doses greater than approximately 800,000 Gy suggests that the CMPO degradation product formed at these absorbed doses is different than product formed at lower absorbed doses. 


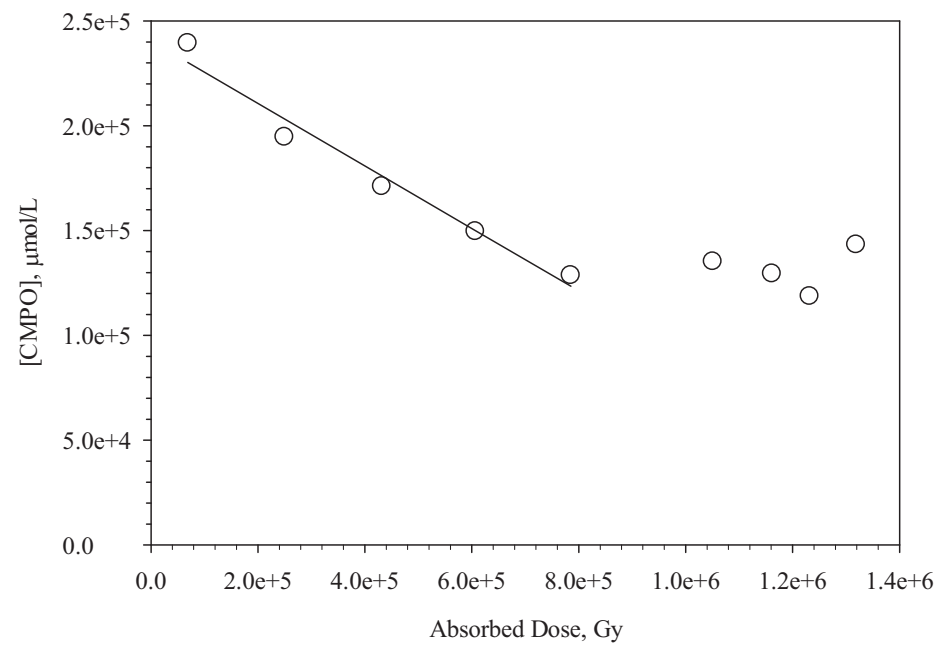

Figure 21. Plot of the concentration of CMPO versus absorbed dose for TRUEX solvent irradiated in contact with 4.4 $\mathrm{M} \mathrm{HNO}_{3}$. The slope of the linear best-fit line corresponds to the $G$-value for the decomposition of CMPO. The determined $G$-value for the destruction of CMPO is $-G_{\mathrm{CMPO}}=-0.159 \pm$ $0.016 \mu \mathrm{mol} \cdot \mathrm{L}^{-1} \cdot \mathrm{Gy}^{-1} \cdot\left(\mathrm{R}^{2}=0.97\right)$

Mincher and co-workers ${ }^{13}$ have measured the $G$-value for CMPO degradation using 0.1 M CMPO in ndodecane. The $G$-value corresponding to CMPO degradation for an organic only irradiation is 0.14 $\mu \mathrm{mol} \cdot \mathrm{L}^{-1} \cdot \mathrm{Gy}^{-1}$. When TRUEX solvent was irradiated in contact with $4.4 \mathrm{M}$ nitric acid in the INL Test Loop the $G$-value for CMPO degradation is $-G_{\mathrm{CMPO}}=-0.159 \pm 0.016 \mu \mathrm{mol} \cdot \mathrm{L}^{-1} \cdot \mathrm{Gy}^{-1}$. This value agrees with the value reported by Mincher and co-workers, but is significantly higher than the value reported by Nash et al. ${ }^{14}$ of $G_{\mathrm{CMPO}}=0.100 \mu \mathrm{mol} \cdot \mathrm{L}^{-1} \cdot \mathrm{Gy}^{-1}$. This $G$-value for the destruction of CMPO was calculated by assuming the values these authors reported for the production of several CMPO radiolysis products can be summed and treated as a $G$-value for the radiolytic destruction of CMPO. However, all the radiolysis degradation products were likely not measured

In addition, to the determination of CMPO, the HPLC analyses identified at least three unknown radiolysis degradation products (see Figure 19). Elias et al. ${ }^{15}$ have used HPLC with electrospray ionization mass spectrometric (ESI-MS) detection to study the radiolytic degradation of CMPO dissolved in $n$-dodecane. Based upon similarities in the results obtained in that work and those reported here, the peak in the HPLC chromatograph with a retention time, $\mathrm{RT}=8.8 \mathrm{~min}$, is likely the monoisobutyl CMPO radiolytic degradation product of CMPO. Unfortunately, similar HPLC-ESI-MS measurements have not been performed on the irradiated TRUEX solvent. It is hoped that the future HPLC-ESI-MS experiments will be able to confirm the presence of the monoisobutyl CMPO in the Test Loop samples and also identify the other unknown radiolytic degradation products detected by HPLC.

The main degradation product formed via $\gamma$-irradiation of TBP is HDBP. The total concentration of HDBP determined in the organic and aqueous phases is plotted as $\mu \mathrm{mol} / \mathrm{L}$ HDBP versus Gy in Figure 22. Linear regression of the data presented in Figure 22 is used to determine the $G$-value for the production of HDBP, $G_{\mathrm{HDBP}}=0.118 \pm 0.007 \mu \mathrm{mol} \cdot \mathrm{L}^{-1} \cdot \mathrm{Gy}^{-1}$. This $G$-value falls within the range of values reported in the review of TBP radiolysis by Mincher and co-authors ${ }^{1}$ and agrees well with the $G$-value for the destruction of TBP reported in this work. 


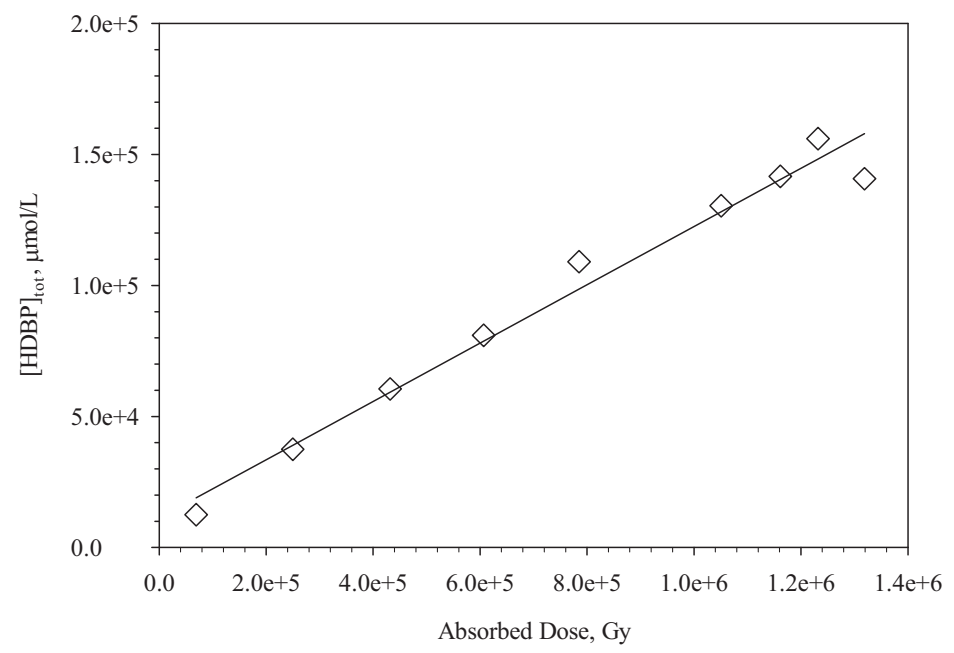

Figure 22. Plot of the total concentration of HDBP present in the aqueous and organic phases versus absorbed dose for TRUEX solvent irradiated in contact with $4.4 \mathrm{M} \mathrm{HNO}_{3}$. The slope of the linear best-fit line corresponds to the $G$-value for the production of HDBP. The determined $G$-value for the overall production of HDBP is $G_{\mathrm{HDBP}}=0.118 \pm 0.007 \mu \mathrm{mol} \cdot \mathrm{L}^{-1} \cdot \mathrm{Gy}^{-1} \cdot\left(\mathrm{R}^{2}=0.97\right)$

The concentration of $\mathrm{H}_{2} \mathrm{MBP}$ determined by IC analysis of the irradiated is plotted in Figure 23 as $\mu \mathrm{mol} / \mathrm{L}$ $\mathrm{H}_{2} \mathrm{MBP}$ versus Gy. Linear regression of the data presented in Figure 23 is used to determine the $G$-value for the production of $\mathrm{H}_{2} \mathrm{MBP}, G_{\mathrm{H} 2 \mathrm{MBP}}=1.56 \times 10^{-3} \pm 8.06 \times 10^{-5} \mu \mathrm{mol} \cdot \mathrm{L}^{-1} \cdot \mathrm{Gy}^{-1}$. The $G$-value for the production of $\mathrm{H}_{2} \mathrm{MBP}$ reported here is rather low. Since the $G$-value for the production of HDBP is 0.118 $\mathrm{mol} \cdot \mathrm{L}^{-1} \cdot \mathrm{Gy}^{-1}$, the expected value for $G_{\mathrm{H} 2 \mathrm{MBP}}$ would be approximately $0.018 \mu \mathrm{mol} \cdot \mathrm{L}^{-1} \cdot \mathrm{Gy}^{-1}$. The lower value of $G_{\mathrm{H} 2 \mathrm{MBP}}$ indicates that processes in addition to radiolysis are likely responsible the destruction $\mathrm{H}_{2} \mathrm{MBP}$.

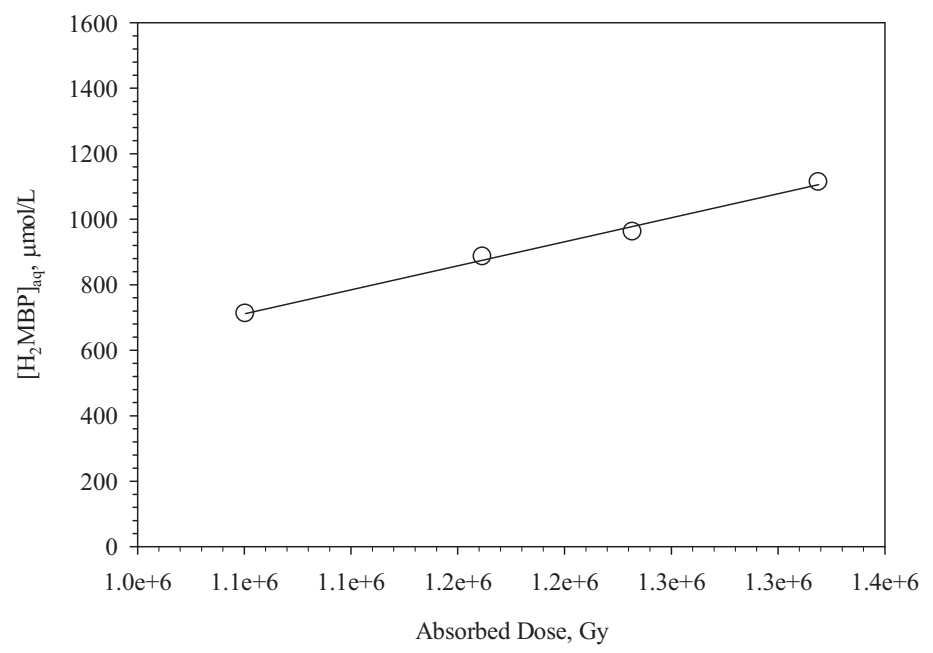

Figure 23. Plot of the concentration of $\mathrm{H}_{2} \mathrm{MBP}$ present in the aqueous phase versus absorbed dose for TRUEX solvent irradiated in contact with $4.4 \mathrm{M} \mathrm{HNO}_{3}$. The slope of the linear best-fit line corresponds to the $G$-value for the production of $\mathrm{H}_{2} \mathrm{MBP}$. The determined $G$-value for the production of $\mathrm{H}_{2} \mathrm{MBP}$ is $G_{\mathrm{H} 2 \mathrm{MBP}}=1.56 \times 10^{-3} \pm 8.06 \times 10^{-5} \mu \mathrm{mol} \cdot \mathrm{L}^{-1} \cdot \mathrm{Gy}^{-1} \cdot\left(\mathrm{R}^{2}=0.99\right)$ 
The concentration of phosphate anion determined by IC analysis of the irradiated is plotted in Figure 24 as $\mu \mathrm{mol} / \mathrm{L} \mathrm{PO}_{4}^{-3}$ versus Gy. Linear regression of the data at presented in Figure 24 is used to determine the $G$-value for the production of $\mathrm{PO}_{4}^{-3}$ anion, $G_{\mathrm{PO} 4-3}=5.60 \times 10^{-3} \pm 3.94 \times 10^{-4} \mu \mathrm{mol} \cdot \mathrm{L}^{-1} \cdot \mathrm{Gy}^{-1}$. Only the somewhat linear portion of the data at absorbed doses greater than approximately $60000 \mathrm{~Gy}$ is used in the linear regression analysis. Limiting the data used to determine the G-value for the production of phosphate anion is justified by the fact that phosphate product at lower dose is limited by the small concentration of HDBP and H2MBP produced at these lower absorbed doses (see Figures 22 and 23).

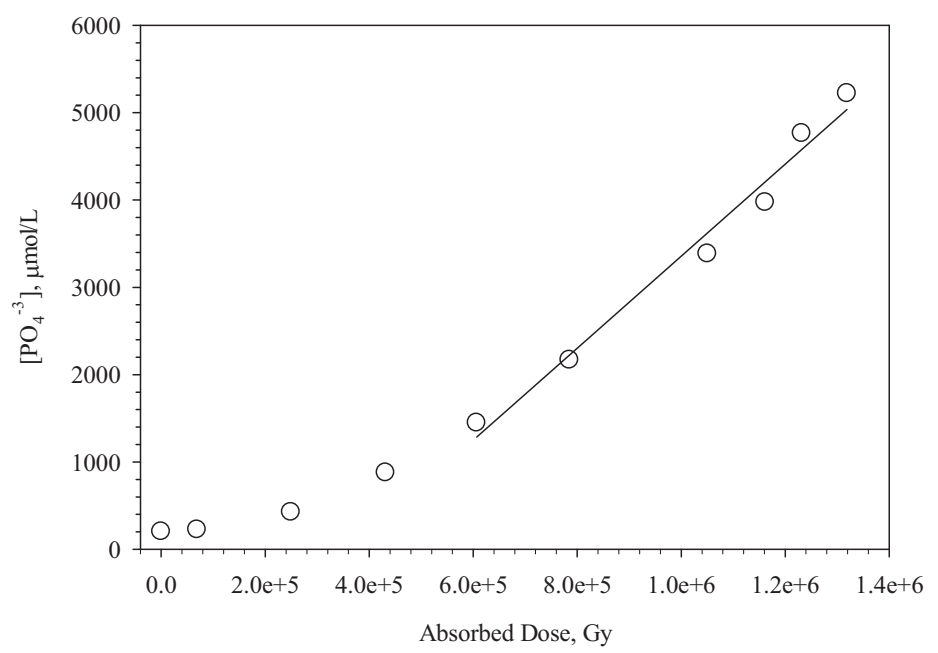

Figure 24. Plot of the concentration of phosphate anion present in the aqueous phase versus absorbed dose for TRUEX solvent irradiated in contact with $4.4 \mathrm{M} \mathrm{HNO}_{3}$. The slope of the linear best-fit line corresponds to the $G$-value for the production of phosphate anion. The determined $G$-value for the production of phosphate is $G_{\mathrm{PO} 4-3}=5.60 \times 10^{-3} \pm 3.94 \times 10^{-4} \mu \mathrm{mol} \cdot \mathrm{L}^{-1} \cdot \mathrm{Gy}^{-1} \cdot\left(\mathrm{R}^{2}=0.98\right)$

Since the direct production of phosphate anion from with TBP or HDBP is not a process likely to occur, the phosphate anion must be produced via decomposition of $\mathrm{H}_{2} \mathrm{MBP}$. The $G$-value for the production of $\mathrm{PO}_{4}^{-3}$ anion reported here is higher than the $G$-value determined for the production of $\mathrm{H}_{2} \mathrm{MBP}$ in this work. This higher value indicates that either a process in addition to radiolysis is responsible for the production of phosphate anion or that the rate of radiolytic degradation of $\mathrm{H}_{2} \mathrm{MBP}$ to form phosphate anion is significantly greater than the rate of radiolytic degradation of HDBP to form $\mathrm{H}_{2} \mathrm{MBP}$.

\section{Conclusions and Continuing Investigations}

The INL radiolysis and hydrolysis test loop has been used to evaluate the effects of hydrolytic and radiolytic degradation upon the efficacy of the TRUEX flowsheet for the recovery of trivalent actinides and lanthanides from acidic solution. The results demonstrate that solvent hydrolysis does not adversely impact the performance of the process flowsheet.

The nominal composition of the TRUEX solvent used in this study is $0.2 \mathrm{M} \mathrm{CMPO}$ and $1.4 \mathrm{M}$ TBP dissolved in n-dodecane. Gamma irradiation of a mixture of TRUEX process solvent and nitric acid in the test loop does adversely impact flowsheet performance as measured by the decreasing americium and europium distribution ratios in the extraction section as accumulated dose increases. The observed increase in americium and europium stripping distribution ratio with increasing absorbed dose indicates the radiolytic production of organic soluble degradation compounds. These organic soluble degradation products inhibit stripping of the irradiated TRUEX solvent with dilute nitric acid. Using GC, IC and 
HPLC, the changes in the TRUEX solvent composition were quantified. The changes in the TRUEX solvent composition correlate will with variation in the measured extraction, scrub, and strip Am and Eu distribution ratios.

Operation of the TRUEX flowsheet would require careful monitoring to ensure extraction distributions are maintained at acceptable levels. Results of flowsheet testing demonstrate that acidic degradation products are removed from the solvent. Non-acidic degradation products build-up in the process solvent but these products do not adversely impact stripping performance. Use of an aqueous complexant in the aqueous strip solution easily overcomes the adverse impacts of non-acidic degradation and maintains very efficient stripping of the TRUEX process solvent at all absorbed doses studied.

Continuing work with will focus on the further development and optimization of the HPLC-ESI-MS methods used for the identification of degradation products produced by $\gamma$-irradiation of the TRUEX process solvent. Subsequent research will evaluate the mechanism of degradation product formation in the strip section of TRUEX process flowsheet.

\section{Acknowledgements}

The authors wish to thank Cathy Rae for assistance with the GC analyses. The authors, also, thank Bruce J. Mincher for the many useful discussions of radiation chemistry in aqueous and organic solutions. 


\section{References}

1. Mincher, B. J.; Modolo, G.; Mezyk, S. P. Solvent Extr. Ion Exch. 2009, 27, 1 - 25.

2. Mincher, B. J.; Modolo, G.; Mezyk, S. P. Solvent Extr. Ion Exch. 2009, 27, 579 - 606.

3. Buxton, G. V.; Greenstock, C. L.; Helman, W. P.; Ross, A. B. J. Phys. Chem. Ref. Data 1988, 17, 513 $-866$.

4. Mincher, B. J.; Mezyk, S. P. Radiochim. Acta 2009, 97, 519 - 534.

5. Mincher, B. J. An overview of selected radiation chemical reactions affecting fuel cycle solvent extraction. Ch. 15 in: Nuclear Energy and the Environment, ACS Symposium Series 1046, Wai, C. M.; Mincher, B. J., Eds. 2010.

6. Peterman, D. R.; Garn, T. G.; Herbst, R. Scott; Law, J. D.; Meikrantz, D. H.; Tillotson, R. D. Completion of Design and Installation of Radiolysis/Hydrolysis Test Loop, Idaho National Laboratory, AFCI-SEPA-PMO-MI-DV-2009-000122, July 23, 2009.

7. Black, T. H. Aldrichimica Acta 1983, 16, 3 - 10.

8. Dodi, A.; Verda, G. J. Chromat. A 2001, 920, 275 - 281.

9. Sehested, H. The Fricke Dosimeter, in Manual on Radiation Dosimetry, Marcel Dekker, Inc. Holm, N. W.; Berry, R. J., Eds. 1970, $313-317$.

10. Ajji, Z. Rad. Measure. 2006, 41, $438-442$.

11. Peterman, D. R.; McDowell, R. G.; Olson, L. G.; Tillotson, R. D. "FY-2011 Status Report for the Radiolysis and Hydrolysis Test Loop," Idaho National Laboratory, FCR\&D-SWF-2011-000286, September 9, 2011.

12. Peterman, D. R.; Mincher, B. J.; Elias, G.; Tillotson, R. D.; Riddle, C. L. FY-2010 Status Report for the Radiolysis and Hydrolysis Test Loop, Idaho National Laboratory, FCR\&D-SEPA-2010-000185, September 29, 2010.

13. Mincher, B. J.; Mezyk, S. P.; Groenewold, G.; Elias, G. A Comparison of the Alpha and Gamma Radiolysis of CMPO, Idaho National Laboratory, FCRD-SEPA-2011-000148, June 15, 2011.

14. Nash, K. L.; Rickert, P. G.; Horwitz, E. P. Solvent Extr. Ion Exch. 1989, 7, 655 - 675.

15. Elias, G.; Groenewold, G. S.; Mincher, B. J.; Mezyk, S. P. J. Chromat. A, manuscript submitted. 